\title{
Fluid-structural design analysis for composite aircraft wings with various fiber properties
}

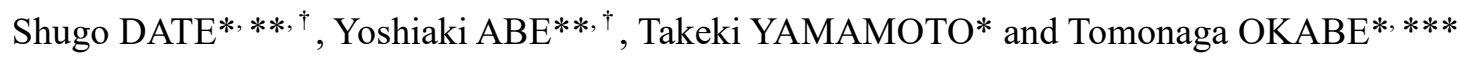 \\ *Department of Aerospace Engineering, Tohoku University \\ 6-6-01, Aramaki-Aza-Aoba, Aobaku, Sendai, 980-8579, Japan \\ ${ }^{* *}$ Institute of Fluid Science, Tohoku University \\ 2-1-1, Katahira, Aobaku, Sendai, 980-8577, Japan \\ E-mail: yoshiaki.abe@tohoku.ac.jp \\ ${ }^{* * *}$ Department of Materials Science and Engineering, University of Washington \\ BOX 352120, Seattle, WA 98195, U.S.A. \\ 'Shugo DATE and Yoshiaki ABE contributed equally to this work.
}

Received: 23 March 2020; Revised: 2 August 2020; Accepted: 16 September 2020

\begin{abstract}
This study performed an analysis for the fluid-structural design of aircraft wings composed of carbon fiber reinforced plastics (CFRPs). Specifically, the effects of carbon fibers on structural weight were evaluated. A multiscale computational framework was developed for designing CFRP wings so that even those CFRPs can be considered whose mechanical properties are not available as experimentally-measured data, thereby bridging two different scales by the following processes: 1) a microscale analysis for evaluating the mechanical properties (stiffness and strength) of unidirectional CFRP laminates and 2) a macroscale fluid-structural analysis that involves structural sizing of wingbox structures based on the mechanical properties given by the microscale analysis. To this end, five fibers were examined in this study, namely: T300, T700S, T800H, T800S, and T1100G. It was discovered that $\mathrm{T} 1100 \mathrm{G}$ exhibited the lightest wingbox structures, followed by T800S, T800H, T700S, T300. This was mainly due to the difference in a thickness of the lower panels, where the thickness was minimized with T1100G among the five fibers, resulting from the tensile failure mode. Meanwhile, the upper panels under compressive load showed two different failure modes, namely: fiber microbuckling and skin buckling. In the region where the fiber microbuckling was dominant, the panel thickness was in order of the stiffness of the fiber, i.e., the panel made with T1100G having the highest stiffness was thicker than that made with T800S, T800H, T700S and T300, and vice versa in the region where the skin buckling was dominant. Based on the microscale analysis, the aforementioned failure mechanisms are consistent with the fact that a quasiisotropic laminate with the fibers of higher stiffness is more resistant to tensile load and skin buckling but less resistant to compressive load.
\end{abstract}

Keywords : Fluid-structure interaction, Composite materials, Multiscale modeling, Aeroelastic analysis, Aircraft wing, Computational Fluid Dynamics

$\begin{array}{ll}\text { Nomenclature } & \\ E & =\text { Young's modulus } \\ G & =\text { shear modulus } \\ v & =\text { Poisson's ratio } \\ \sigma_{0} & =\text { Weibull scale parameter of fiber strength } \\ m & =\text { Weibull scale modulus of fiber strength } \\ \sigma_{\mathrm{y}} & =\text { yield stress of matrix } \\ \theta & =\text { sweep back angle } \\ c_{1} & =\text { chord length at wing root } \\ c_{2} & =\text { chord length at kink }\end{array}$




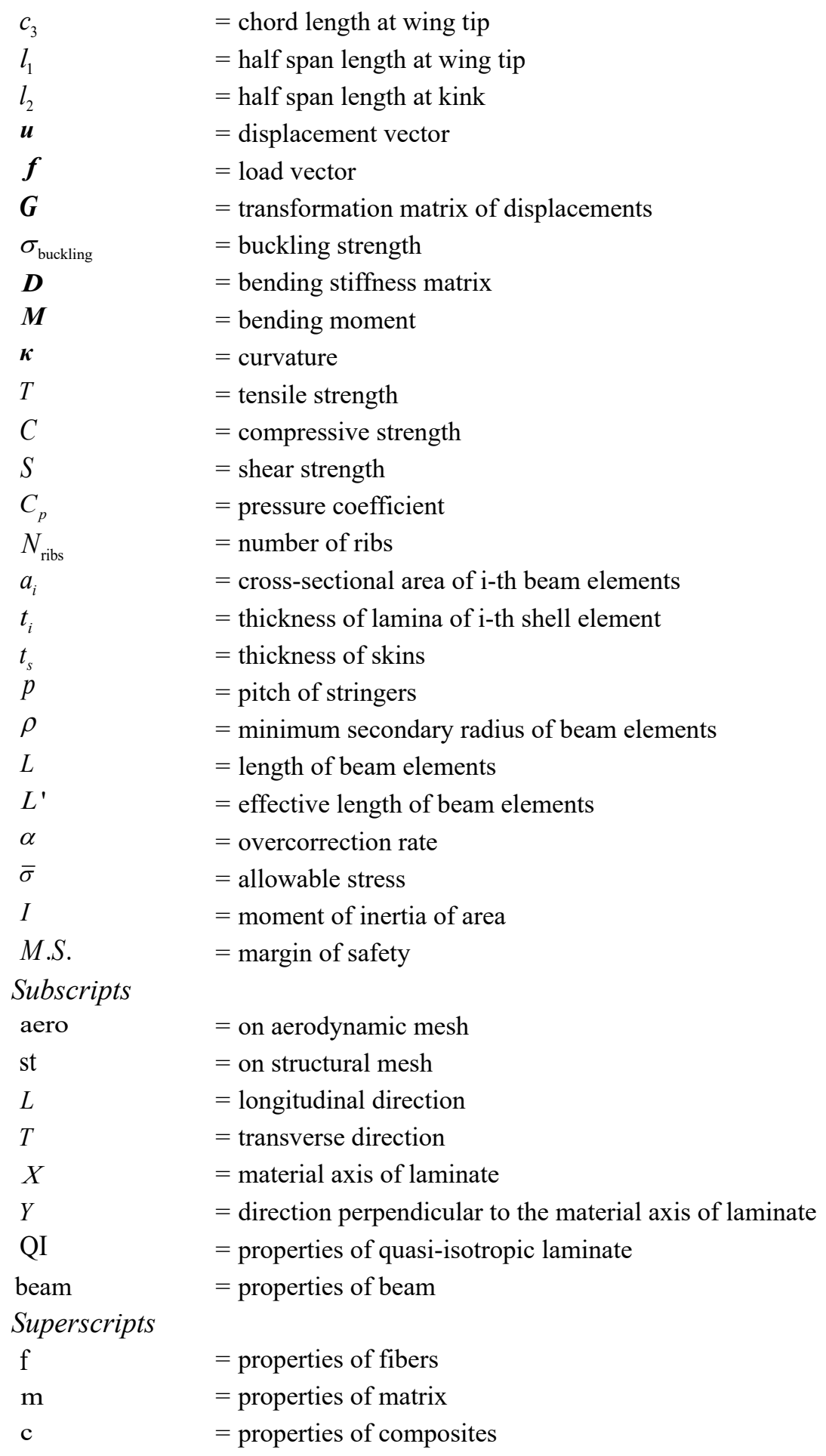

\section{Introduction}

A carbon fiber reinforced plastic (CFRP) is a composite material composed of resin and carbon fiber. CFRPs comprise specific strengths and stiffnesses that are superior to those of metal-based materials, enabling the fabrication of lighter and stronger structures. In structural applications, CFRPs are used to fabricate laminate materials wherein unidirectional laminates are oriented at various angles. There is a high degree of freedom in designing a CFRP; the fibers and resins used in the material can be freely selected, and various advantages can be realized by changing the orientation angle of the laminate. In recent years, these materials have been used in the construction of the structural members of transport aircrafts, of which approximately $50 \%$ are now composed of CFRPs. 
However, the complexity of the CFRP microstructure has impeded its utilization in further applications. A rather significant problem is that the fibers and resins tend to exhibit different mechanical properties. Specifically, Young's moduli of the carbon fibers and resins used in CFRPs can differ by a factor of up to 100, which can lead to complex stress fields in the resin as a result of fiber constraints. Accordingly, the failure mechanism of a CFRP is bound to be fundamentally dependent on the load direction. Furthermore, the fact that CFRPs have high degrees of design freedom in terms of the stacking sequence, renders the design of a structure composed of CFRPs more complicated. For example, under a tensile strain in the fiber direction, breakage occurs as a result of fiber breakage; whereas, kink bands have been observed to form under a compression in the fiber direction. At the same time, the resulting tension and compression in a direction perpendicular to the fiber lead to the generation of cracks within the resin, which ultimately propagate to cause a fracture.

These complex failure mechanisms can significantly hinder the design of CFRP-based aircraft components; therefore, these materials are affected by several unresolved design challenges and have not demonstrated sufficient potential for weight reduction. The aircraft design process can be divided into three sub-processes: conceptual, basic, and detailed design. In the conceptual design process, body shapes and features are determined to meet design requirements, and schematics of the shapes are drawn as trihedral figures. In the basic design process, more detailed shapes are developed through numerical analysis, wind tunnel testing, and structural design. At the end of this process, structural materials are selected for the design of structural members, and the aircraft specifications are determined. In the detailed design process, the actual parts are manufactured and assembled based on the information defined in the previous stage. Strength and fatigue testing at the component level is performed in this stage. Based on the test results, the basic and detailed design processes are repeated until satisfactory results are achieved. Variations in the material type, such as the use of CFRPs instead of duralumin, have a significant impact on the structural design process; whereas, the aerodynamic design is affected by changes in the aerodynamic characteristics caused by such structural changes. Therefore, CFRPs with complex failure mechanisms can prolong the process of aircraft designing. Thus far, aircraft design tools that take into account the failure mechanisms of CFRPs have not been developed in industrial application levels; consequently, the development of a simulation tool capable of designing aircraft while considering the complexities of CFRP materials is highly desired (Baker and Scott, 2004).

Research on aircraft design and weight estimation using composite materials has been progressing for decades. Recent advances in computational architecture and associated software technologies have enabled the fully-simulated shape optimization of aircraft wings. Obayashi et al. (2007) applied the genetic algorithm (GA) to implement an evolutionary method for wing shape optimization. Their work considered the drag on the wing in both supersonic and subsonic conditions wherein the bending moment at the wing root was used as the objective function, which was estimated using the computational fluid dynamic (CFD) code. Their approach has already been applied in the design of regional jets, although the use of CFRPs was not considered in these simulations. Kennedy and Martins (2012) proposed an optimization framework based on an adjoint method involving high-fidelity fluid-structural coupled analysis. By optimizing the wing of a common research model (Lyu, et al., 2015), they obtained the optimal wing structure thicknesses and wing shapes. Their results successfully demonstrated the advantage of composites as compared to conventional metallic materials in terms of and the takeoff gross-weight and fuel burn. Their framework, however, begins from a macroscopic analysis based on the known mechanical properties of CFRPs, and thus the individual effects of fiber and resin properties on the final aerostructural design of composite wings were not clarified; this is required for evaluating the use of new CFRPs with unknown mechanical properties. In contrast, Llorca et al. (2011) proposed a multiscale modeling strategy to carry out high-fidelity virtual mechanical testing of composite materials and structures. Their approach begins with an in-situ measurement of the matrix and interface mechanical properties at the nanometermicrometer range, from which a ladder of numerical simulations from the nanometer to millimeter scales is carried out. However, the concept of their work has not been applied to practical engineering problems, including aircraft wing designs, which are the focus of this study. Practically, computational evaluations of mechanical properties will be required when considering CFRP laminates, for which the mechanical properties have not yet been experimentally measured; this is because the additional experimental measurements often lead to an increase in the development cost. As such, the development of a framework has not been achieved for designing CFRP wings based on a multiscale analysis, which initiates from the evaluation of mechanical properties at the level of fibers and resin.

The purpose of this study is to investigate the effects of mechanical properties of the fibers on the performance of CFRP wings, especially on wing weight. Therefore, assuming that the wing shape (planform and airfoil shape) is fixed, 
different combinations of fibers (T300, T700S, T800H, T800S and T1100G) and resin (\#3900-2B) are examined for structural sizing of CFRP wings. Note that the mechanical properties of CFRPs (T300/\#3900-2B, T700S/\#3900-2B, T800H/\#3900-2B, T800S/\#3900-2B and T1100G/\#3900-2B) have not been reported in the literature, some of which is reported for the first time herein based on the microscale analysis explained later. A new framework for designing composite aircraft wings was developed for this purpose, which was based on a multiscale analysis; this framework ranged from the microscale, such as the fibers and resins in the composite material, to the macroscale, such as the aerostructural analysis for wing box structures. Based on this framework, fluid-structural analysis of aircraft wings with various fibers was carried out to evaluate the effects of fibers on the weight of the wingbox structure. The remainder of this paper is organized as follows. Section 1 introduces the background of the study. Section 2 provides a problem specification. Section 3 describes several methods used in the multiscale framework, and Section 4 discusses computational results. Finally, Section 5 concludes this paper.

\section{Problem specification}

\subsection{Material}

Figure 1 shows the physical properties of various fibers, each having characteristics such as high rigidity and high strength.

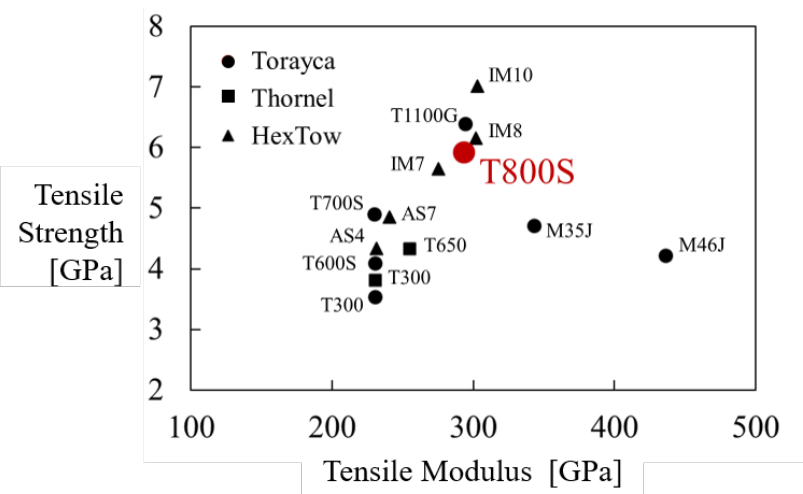

Fig. 1 Plot of strength vs modulus for currently used carbon fibers (Baker and Scott, 2004; TORAYCA, 2020a, 2020b).

In our analysis, T800S is mainly considered because it is being applied to actual machines as aircraft-grade fiber, e.g., for B787s and B777s. We compared T300, T700S, T800H, T800S and T1100G, which had Young's moduli and strengths differing from those of T800S (manufactured by TORAYCA). The physical properties used in the analysis are summarized in Table 1, where $E^{\mathrm{f}}, G^{\mathrm{f}}$, and $v^{\mathrm{f}}$ represent Young's modulus (Watanabe et al., under review), shear modulus, and Poisson's ratio, respectively, and the subscripts $L$ and $T$ represent the longitudinal direction and the transverse direction, respectively. To determine the Young's modulus in the direction perpendicular to the fibers and Poisson's ratio, we referred to the properties of T700G and T800S in Yoshioka et al. (2016) as the properties of T700S (T300) and T800S $(\mathrm{T} 800 \mathrm{H})$. As shown in Table 1, the Young's modulus in the direction perpendicular to the fibers of T1100G was increased as the Young's modulus in the fiber direction increases. The Poisson's ratio of T1100G was fixed to the values of T700S and T800S. The shear modulus of the fibers was assumed to be $37 \mathrm{GPa} . \sigma_{01}, \sigma_{02}, m_{1}$ and $m_{2}$ indicate the coefficients of the bimodal Weibull distribution of the fiber strength (Watanabe et.al., under review). The diameter of fibers is fixed to be $7 \mu \mathrm{m}$ for simplicity although the properties of composite materials are affected by the diameter of fibers. In each case, the mechanical properties of \#3900-2B in Yoshioka et al. (2016) were used for the material properties of resin, which are shown in Table 2. It is assumed that these properties of \#3900-2B, which is heterogeneous material, are homogenized values since those values are used to compare numerical simulations to tensile tests of specimens in (Yoshioka et al., 2016). 
Table 1 Material properties of fibers used in this study.

\begin{tabular}{c|c|c|c|c|c|c|c|c|c|c}
\hline \hline & $E_{L L}^{\mathrm{f}}[\mathrm{GPa}]$ & $E_{T T}^{\mathrm{f}}[\mathrm{GPa}]$ & $G_{L T}^{\mathrm{f}}[\mathrm{GPa}]$ & $G_{T T}^{\mathrm{f}}[\mathrm{GPa}]$ & $v_{L T}^{\mathrm{f}}$ & $v_{T T}^{\mathrm{f}}$ & $\sigma_{01}[\mathrm{GPa}]$ & $\sigma_{02}[\mathrm{GPa}]$ & $m_{1}$ & $m_{2}$ \\
\hline $\mathrm{T} 300$ & 230 & 17.5 & 37 & 37 & 0.17 & 0.46 & 4.1 & 4.2 & 4.0 & 15 \\
$\mathrm{~T} 700 \mathrm{~S}$ & 230 & 17.5 & 37 & 37 & 0.17 & 0.46 & 5.2 & 6.1 & 4.8 & 12 \\
$\mathrm{~T} 800 \mathrm{H}$ & 294 & 19.5 & 37 & 37 & 0.17 & 0.46 & 5.9 & 6.3 & 7.5 & 13 \\
$\mathrm{~T} 800 \mathrm{~S}$ & 294 & 19.5 & 37 & 37 & 0.17 & 0.46 & 6.9 & 8.3 & 4.1 & 13 \\
$\mathrm{~T} 1100 \mathrm{G}$ & 324 & 20.5 & 37 & 37 & 0.17 & 0.46 & 7.7 & 9.1 & 4.5 & 13 \\
\hline
\end{tabular}

Table 2 Material properties of resin used in this study.

\begin{tabular}{c|c|c|c}
\hline \hline & $E^{\mathrm{m}}[\mathrm{GPa}]$ & $v^{\mathrm{m}}$ & $\sigma_{\mathrm{y}}[\mathrm{MPa}]$ \\
\hline$\# 3900-2 \mathrm{~B}$ & 3.2 & 0.38 & 75.0 \\
\hline
\end{tabular}

\subsection{Wing and airflow configuration}

This section describes the configuration of aircraft wing and its design variables. Figure 2 shows a planform of the aircraft wing developed in this analysis. Here, $\theta$ is the sweep angle; $c_{1}$ is the chord length of the wing root; $c_{2}$ is the chord length of the kink; $c_{3}$ is the chord length of the wing tip; $l_{1}$ is the half-span length; and $l_{2}$ is the length to the kink. The airfoil was set as RAE2822, which is one of the typical transonic airfoils. The planform of the wing was determined in reference to information on the Boeing 777 aircraft, of which parameters are listed in Table 3. In addition, the dihedral angle and the torsion-down angle at the wing tip were set to be 4 and 3 deg., respectively.

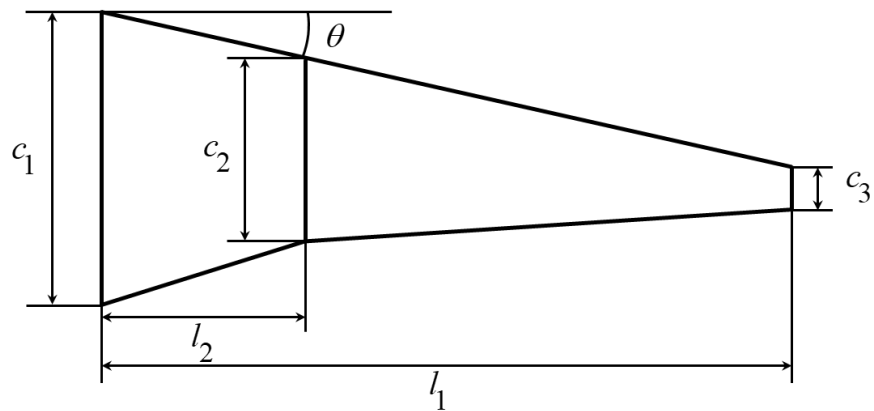

Fig. 2 Definition of planform parameters used in this study.

Table 3 Parameters of wing planform.

\begin{tabular}{|c|c|c|c|c|c|}
\hline$\theta \quad$ [deg] & $c_{1}[\mathrm{~m}]$ & $c_{2} \quad[\mathrm{~m}]$ & $c_{3}[\mathrm{~m}]$ & $l_{1}[\mathrm{~m}]$ & $l_{2}[\mathrm{~m}]$ \\
\hline 34 & 13.081 & 5.969 & 1.778 & 27.94 & 7.366 \\
\hline
\end{tabular}

The structure of the wingbox inside the aircraft wings, which bears a substantial percentage of the load applied to the wing, was then developed. In this case, a multi-stringer stress skin construction with a double spar for use in medium and large passenger aircraft was designed. The stringers were placed on the skin at intervals of $0.2032 \mathrm{~m}$, while the two spars were arranged at positions 20 and $65 \%$, respectively, from the leading edge. The ribs were arranged parallel to the flow direction at the center wing; following the kink, they became perpendicular to the leading edge. A total of 37 ribs were used. Figure 3 shows the wing shape and corresponding finite element model. In this study, skins, ribs and spar webs are modeled by shell (MITC4) elements while spar flanges are modeled by general curved beam elements (Dvorkin and Bathe, 1984; Bathe, 2006). The shell elements used in this study are laminated shell elements, and the stacking sequence is fixed to be [0/45/-45/90 $]_{\text {sym }}$. as shown in Fig. 4. For the Young's modulus of the beam elements, the stiffness of the quasi-isotropic laminates was determined by the classical laminate theory. In this study, it is assumed that the thicknesses and areas of the structural elements are fixed in the chordwise direction. The design variables for the finite element model are summarized in Table 4. Note that, although the wing weight is influenced by both the size of structural elements and structural layouts, this study considers only the size of structural elements as a starting point. Further investigation on optimization of structural layouts will be left to the future study. 


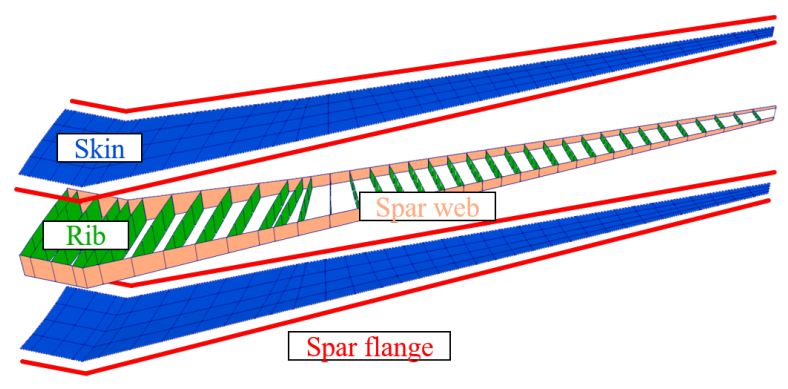

Fig. 3 Finite element model of wings composed of beam and shell elements.

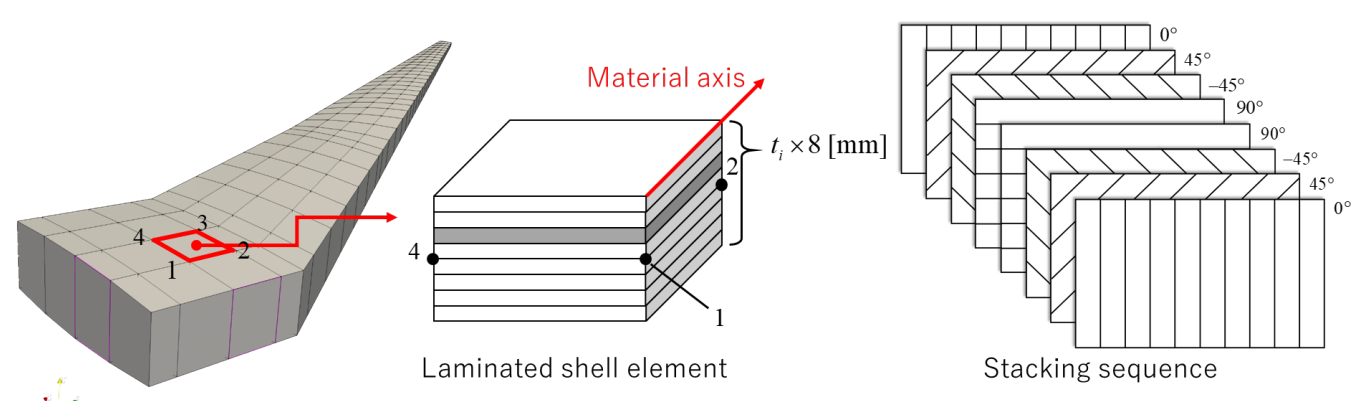

Fig. 4 Definition of laminated shell elements.

Table 4 List of design variables for structure.

\begin{tabular}{c|l|l}
\hline \hline Fixed variables & Description & Values \\
\hline$N_{\text {ribs }}$ & Number of ribs & 37 \\
$p$ & Pitch of stringers & $0.2032 \mathrm{~m}$ \\
\hline \hline Design variables & Description & Minimum - Maximum values \\
\hline$t_{1}-t_{\text {nshell }}$ & Thickness of lamina for panels & $0.2032 \mathrm{~mm}-$ No limit \\
$a_{1}-a_{\text {nbeam }}$ & Cross section of beam elements & $2.54-2540 \mathrm{~mm}^{2}$ \\
\hline
\end{tabular}

\section{Methodology}

\subsection{Overview}

In this section, the multiscale analysis approach used in this study is explained. Figure 5 illustrates a schematic flowchart of the methodology. The overall procedure is divided into two stages: macroscale and microscale analysis. The orange section of the figure represents the macroscale analysis. This component involves the application of CFD, computational structural dynamics (CSD), and an interpolation between the CFD and CSD, along with failure analyses and a sizing process. The margin of safety (M.S.) is adopted to define failure criteria and perform structural sizing. In specific, the convergence of structural sizing is defined so that M.S. for all of structural elements are in the range of 0.45 to 0.55 . The blue section represents the microscale analysis involving the estimation of the CFRP mechanical properties. This process includes four independent sub-analyses to predict each property. The material properties estimated by the microscale analysis are used for the failure criteria in the macroscale structural analysis colored in orange in Fig. 4. Each component will be explained in detail from the next section. 


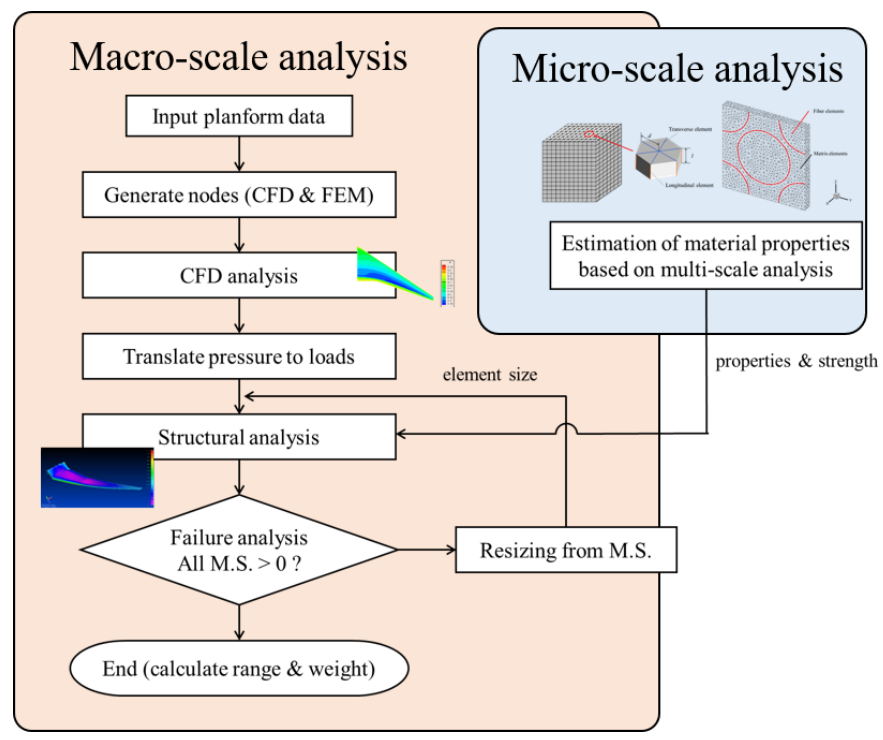

Fig. 5 Overview of the numerical method to design composite aircraft wings with variable fiber and resin properties.

\subsection{Microscale analysis}

\subsubsection{Overview}

This section describes methodologies in the microscale analysis for estimating mechanical properties of the CFRP laminates. The microscale analysis consists of four independent analyses. First, periodic unit cell (PUC) (Okabe, et al., 2015) analysis based on a finite element analysis (FEA) is performed to predict the mechanical properties of the unidirectional laminate. Second, the spring element model (SEM) (Okabe, et al., 2005) is used to estimate the longitudinal tensile strength of the unidirectional laminate. After that, the microbuckling model proposed by Sun and Jun (1994) is applied to estimate the longitudinal compressive strength of the unidirectional laminate. Finally, microscopic FEA using PUC is performed to predict the tensile and compressive strengths in the transverse direction and the in-plane shear strength of the unidirectional laminate.

\subsubsection{Mechanical properties}

Although CFRP is a heterogeneous material composed of fiber and resin, its structural analysis is conducted assuming homogenized physical properties developed from those of unidirectional laminates. For materials such as composites with periodic microstructures, physical properties are estimated using a homogenization method. In this study, we performed PUC analysis (Okabe, et al., 2015) based on a finite element approach, where the mechanical properties of various composite materials are estimated from the properties of their constituent fibers and matrix resin. The fiber volume fraction $V_{f}$ was fixed to be 0.56 , and the estimated mechanical properties of unidirectional composite ( $E_{L L}^{\mathrm{c}}$, $\left.E_{T T}^{\mathrm{c}}, G_{L T}^{\mathrm{c}}, G_{T T}^{\mathrm{c}}, v_{L T}^{\mathrm{c}}, v_{T T}^{c}\right)$ are given in Table 5 .

\subsubsection{Longitudinal tensile strength}

To predict the tensile strength of a composite in the fiber direction, SEM, which was basically proposed by Okabe et al. (2005) and recently developed by Yamamoto et al. (2019) to include the additional stress concentration on the fiber surface, was used in this study. In this model, the fibers in the unidirectional laminates are treated as hexagonally arranged fiber direction spring elements, while the resin is treated as a transverse direction spring element having only shear stiffness.

After the calculation with 100 patterns of random distributions of fiber strength, the weakest tensile strength is used as the longitudinal tensile strength of the unidirectional composite $\left(T_{L L}\right)$; this is given below in Table 6 .

\subsubsection{Longitudinal compressive strength}

In this study, the microbuckling model which considers nonlinearity of resin (Sun and Jun, 1994) was adopted to estimate the longitudinal compressive strength. The longitudinal compressive strength of unidirectional composite $\left(C_{L L}\right)$ is represented in Table 6. 


\subsubsection{Transverse strength and in-plane shear strength}

The PUC simulation (Okabe, et al, 2015) can be also used to determine the tensile and compressive strengths in the transverse direction and the in-plane shear strength of the unidirectional laminate. It is assumed that the fiber is a rectangular anisotropic elastic body with in-plane isotropic properties, that the resin is an isotropic visco-elasto-plastic body. The transverse strength and in-plane shear strength are represented as $\left(T_{T T} C_{T T}, S_{L T}\right)$ in Table 6.

\subsection{Macroscale analysis}

\subsubsection{Aerodynamic analysis}

To analyze aerodynamic forces acting on the wing, the compressible Euler equations are solved in this study. The finite-volume method with an upwind-biased third-order MUSCL scheme (Bram, 1979) was applied, and the minmod limiter function was used to maintain numerical stability. The minnod limiter function had the compressibility parameter of 4.0. The Lower-Upper Symmetric-Gauss-Seidel (Yoon and Jameson, 1988) method was used to carry out implicit time integration for time advancement. The computational grid is a structured grid with the number of $177 \times 80 \times 30$ (freestream, spanwise, and wall-normal directions), and the outer boundaries are located at 30 times the chord length away from the leading edge of the wing root. The flow is solved until it reaches a steady state, and the steady state solution of the pressure distribution on the wing surface was used to evaluate the aerodynamic load. In this study, a compressible inviscid flow was assumed for suppressing a computational cost, which is regarded mostly sufficient for predicting mean aerodynamic performances at transonic cruise conditions (Martins et al., 2001, Du, et al., 2017) although there have been some remarks on the nonnegligible viscous effect for more precise aerodynamic predictions which are often required in the wing-shape optimizations (Kenway 2014).

\subsubsection{Interpolation between CFD and CSD}

In this section, we explain a method for interpolating load from a mesh used in CFD analysis to nodes used in CSD analysis. The aerodynamic load which was interpolated to the structural grid was one-way coupled in the fluid-structure interaction (FSI) viewpoint (Feripa et al., 2001). The load interpolation can be expressed as a transposition of the matrix $\boldsymbol{G}$ used for the interpolation of displacements from structural mesh to aerodynamic mesh.

The displacement obtained from the mesh used for the CSD analysis was interpolated to the mesh used in the CFD analysis via a Constant Volume Transformation (CVT) method (Goura, 2001) as in Eq. (1).

$$
\boldsymbol{u}_{\text {aero }}=\boldsymbol{G} \boldsymbol{u}_{\text {st }},
$$

where $\boldsymbol{u}_{\text {aero }}$ is the displacement of aerodynamic mesh, $\boldsymbol{u}_{\text {st }}$ is the displacement of structural mesh. Using the displacement interpolation matrix $\boldsymbol{G}$ created via the CVT method the interpolation of load vectors is described as follows:

$$
\boldsymbol{f}_{\text {st }}=\boldsymbol{G}^{T} \boldsymbol{f}_{\text {aero }},
$$

where $\boldsymbol{f}_{\text {st }}$ is the load vectors on structural mesh and $\boldsymbol{f}_{\text {aero }}$ is the load vectors on aerodynamic mesh. Note that, in this study, only the interpolation of the load from the aerodynamic mesh is performed because we performed one-way coupled analysis for an aeroelastic problem.

\subsubsection{Structural analysis}

In this study, linear-elastic FEA was performed to estimate the stress field in wingbox structures experiencing aerodynamic force. Here, load factor of 2.5 and a safety factor of 1.5 were considered, and the vector obtained by multiplying the vector interpolated from the aerodynamic force by 3.75 was used as the external force. The resultant stress field of wingbox structures was used for the failure analysis below to estimate the failure of each structural part.

\subsubsection{Failure analysis}

Two types of constraints are adopted for modeling the failure of CFRP laminates: a local buckling of the skin between stiffeners and a stress failure criterion. Since the laminated shell elements are used for skins, the local buckling constraint is evaluated using the averaged stress for all layers while the stress failure criterion is evaluated by the stress in each layer. Each failure criterion will be incorporated into the structural sizing with non-zero M.S. value in Eq. (15). 
The local buckling of skin is evaluated by two equations below (Kennedy, 2014). Note that again, the averaged stress of all layers is used in the local buckling failure.

$$
\begin{aligned}
& \sigma_{\text {buckling }}=\frac{2 \pi\left(\sqrt{D_{11} D_{22}}+D_{12}+2 D_{66}\right)}{t_{s} p^{2}} \\
& \sigma_{\text {buckling }}=\frac{4}{t_{s} p^{2}}\left(D_{11} D_{22}^{3}\right)^{0.25}\left(8.125+\frac{5.045}{\xi}\right) \quad(\text { If } \xi>1) \\
& =\frac{4}{t_{s} p^{2}} \sqrt{D_{22}\left(D_{12}+2 D_{66}\right)}\left(11.7+0.532 \xi+0.938 \xi^{2}\right) \quad(\text { If } \xi>1) \\
& \xi=\frac{\sqrt{D_{11} D_{22}}}{D_{12}+2 D_{66}}
\end{aligned}
$$

where $\boldsymbol{D}$ is the bending stiffness matrix, which is calculated using classical laminate theory; $t_{\mathrm{s}}$ is the thickness of skins, which is half the thickness of shell elements; and $p$ is the pitch of stringers and is set to be $0.2032 \mathrm{~m}$. The first and second equations represent the buckling strength due to compression and shear, respectively. The bending matrix $\boldsymbol{D}$ is defined as

$$
\left\{\begin{array}{c}
M_{X} \\
M_{Y} \\
M_{X Y}
\end{array}\right\}=\left[\begin{array}{lll}
D_{11} & D_{12} & D_{16} \\
D_{12} & D_{22} & D_{26} \\
D_{16} & D_{26} & D_{66}
\end{array}\right]\left\{\begin{array}{c}
\kappa_{X} \\
\kappa_{Y} \\
\kappa_{X Y}
\end{array}\right\}
$$

where $M$ is bending moment, $\kappa$ is curvature, and $(X, Y)$ is a two-dimensional coordinate system with $X$ being the material axis for each element and $Y$ being the perpendicular to the $X$-axis direction. Since we used the thickness of lamina as the design variable, the calculation of bending stiffness was calculated using the average of stiffness $E_{\mathrm{QI}}$ of the eight layers to prevent overestimation of bending stiffness. Eventually, the failure criteria are defined as

$$
F=\left\{\begin{array}{l}
\frac{\sigma_{X X}}{\sigma_{\text {buckling }}} \leq 1 \\
\frac{\sigma_{X Y}}{\sigma_{\text {buckling }}} \leq 1
\end{array} .\right.
$$

where $\sigma_{X X}$ and $\sigma_{X Y}$ means average stress of laminates in the materials coordinates system.

To consider a stress failure criterion for the fiber-dominant failure, two criteria are considered in this study, which are the Christensen's criterion (Christensen, 1997) and the maximum stress criterion as follows:

$$
\begin{gathered}
F=\left(\frac{1}{T_{L L}}-\frac{1}{C_{L L}}\right) \sigma_{L L}+\frac{\sigma_{L L}^{2}}{T_{L L} C_{L L}}-\frac{1}{4}\left(\frac{1}{T_{L L}}+\frac{1}{C_{L L}}\right)^{2} \sigma_{L L} \sigma_{T T} \leq 1 \quad \text { (Christensen's criterion), } \\
F=\max \left\{\frac{\sigma_{L L}}{T_{L L}},-\frac{\sigma_{L L}}{C_{L L}}\right\} \leq 1
\end{gathered}
$$

The difference of these criteria will be discussed in detail along with the computational results in Sec 4.3. To consider a stress failure criterion for the matrix-dominant failure, the Christensen's criterion (Christensen, 1997) is adopted. The criterion is represented by the following equation:

$$
F=\left(\frac{1}{T_{T T}}-\frac{1}{C_{T T}}\right) \sigma_{T T}+\frac{\sigma_{T T}^{2}}{T_{T T} C_{T T}}+\frac{\sigma_{T L}^{2}}{S_{T L}^{2}} \leq 1 \quad \text { (Christensen's criterion). }
$$

Both fiber- and matrix- dominant failure criteria are calculated in each layer. Equation (6) describes the fiber-dominant failure mode, in which the pure tension force breaks the fibers ( $T_{L L}$ obtained by the SEM), and also the pure compression case, in which the fibers cause microbuckling failure ( $C_{L L}$ obtained by the Sun and Jun model (1994)). By contrast, Eq. (7) corresponds to the failure mode controlled by the resin, in which the critical stresses at which resin cracks occur under pure tensile ( $T_{T T}$ obtained by the PUC analysis), compressive ( $C_{T T}$ obtained by the PUC analysis), and shear ( $S_{L T}$ obtained by the PUC analysis) stresses are applied. In this study, the reduction of strength caused by holes for fasteners is accounted 
for by reducing strength parameters to half their original value. Note that again, the stress values of each layer are used to calculate the equations in the Christensen's criterion. The stress values were calculated using the constitutive low and the strains at the center of each layer which is calculated from the displacement fields obtained by finite element analysis.

For modeling the beam element failure, the Johnson-Euler buckling criterion is considered. With this fracture criterion, the buckling stress criterion is switched according to the slenderness ratio of the beam element as follows:

$$
\sigma_{\text {buckling }}= \begin{cases}\sigma_{\max }\left(1-\frac{\sigma_{\max }}{4 \pi^{2} E_{\mathrm{QI}}}\left(\frac{L^{\prime}}{\rho}\right)^{2}\right) & \left(\left(\frac{L^{\prime}}{\rho}\right) \leq \sqrt{\frac{2 \pi^{2} E_{\mathrm{QI}}}{\sigma_{\max }}}\right) \\ \frac{\pi^{2} E_{\mathrm{QI}}}{\left(\frac{L^{\prime}}{\rho}\right)^{2}} & \left(\left(\frac{L^{\prime}}{\rho}\right)>\sqrt{\frac{2 \pi^{2} E_{\mathrm{QI}}}{\sigma_{\max }}}\right)\end{cases}
$$

where $\sigma_{\max }$ is the stress at the compression yield point; $L$ ' is the effective length; $\rho$ is the minimum secondary radius of the section; and $E_{\mathrm{QI}}$ is the Young's modulus. To determine $\sigma_{\max }$, the compressive strength of the beam element is used. $L$ ' and $\rho$ are represented by the following equations:

$$
\begin{gathered}
L^{\prime}=\frac{L}{\sqrt{2}}, \\
\rho=\sqrt{\frac{I}{a}},
\end{gathered}
$$

where $L$ is the length of the beam element; $I$ is the moment of inertia of area; and $a$ is the cross-sectional area of the beam element. Eventually, the failure criterion of the beam element is defined as

$$
F=\frac{\sigma}{\sigma_{\text {buckling }}} \leq 1 .
$$

The criterion for destruction can be determined based on the material strength of the beam element. The cross0sectional shape of the beam element is assumed to be a beam constructed from a composite material with a laminate structure. The corresponding finite element model can then be rendered so that the stiffness is the same as the isotropic laminate in the axial direction. For the purposes of this study, the material strength of the beam element was defined as the point at which the beam element strength becomes the critical strain in each direction:

$$
\begin{gathered}
T_{\text {beam }}=\frac{T_{T T}}{E_{T T}^{\mathrm{c}}} E_{\mathrm{QI}}, \\
C_{\text {beam }}=\frac{C_{L L}}{E_{L L}^{\mathrm{c}}} E_{\mathrm{QI}},
\end{gathered}
$$

where $T_{T T}$ and $C_{L L}$ are the tensile and compressive strengths, respectively. $E_{\mathrm{QI}}$ is the axial stiffness of the quasi-isotropic laminate and is calculated using the classical laminate theory. Consequently, the failure criterion is defined as

$$
F=\max \left\{\frac{\sigma}{T_{\text {beam }}},-\frac{\sigma}{C_{\text {beam }}}\right\} \leq 1 .
$$

As such, two types of stress criterion are adopted for the fiber-dominant failure in CFRP laminates (shell element): the Christensen's criterion in Eq. (6); and the maximum stress criterion in Eq. (6). The other failure criteria are uniquely defined for each failure mode, namely: the matrix-dominant failure and local skin buckling of CFRP laminates (shell element) in Eqs. (7) and (5) as well as the buckling stress criterion and failure criterion for the beam element in Eqs. (10) and (12). Hence, we will compare two results for each fiber using the fiber-dominant failure criteria in Eq. (6) while using Eqs. (5), (7), (10) and (12) for the other failure criteria. Note that again, failure criteria Eqs. (5), (7), (10) and (12) will be incorporated into the structural sizing with non-zero M.S. values in Eq. (15). 


\subsubsection{Sizing process of structural components}

The fully stressed design method (Lansing, 1971) is applied in this study. The fully stressed design is able to derive the design in which the stress on each structural member assumes one of a set of limited allowable stresses. This design method is advantageous in that it is computationally cheap, and no objective function exists. The function does not necessarily lead to weight minimization but can provide values close to the optimal set. The simplest algorithm is to apply the stress ratio for updating element dimensions as follows:

$$
x_{i}^{k+1}=x_{i}^{k}\left(\frac{\sigma_{i}^{k}}{\bar{\sigma}_{i}^{k}}\right)^{\alpha},
$$

where $x_{i}^{k+1}$ and $\sigma_{i}^{k}$ denote the design variable (thickness of shell elements and cross section of beam elements) and the stress on the $i$-th element during the $k$-th step, respectively; $\bar{\sigma}_{i}^{k}$ indicates the allowable stress on the $i$-th element; and $\alpha$ is the overcorrection rate. The calculation was performed with different $\alpha$ for the shell and beam elements:

$$
\alpha=\left\{\begin{array}{cc}
0.25 & \text { (for shell elements) } \\
0.5 & \text { (for beam elements) }
\end{array} .\right.
$$

M.S. is used to assess the safeness of the aircraft structure, which is defined as follows:

$$
M . S_{\cdot_{i}}^{k}=\frac{1}{F_{i}^{k}}-1 \text {. }
$$

In this study, to update structural elements so that M.S. becomes 0.5 , Eq. (13) is rewritten as follows:

$$
x_{i}^{k+1}=x_{i}^{k}\left(1.5 F_{i}^{k}\right)^{\alpha} \text {. }
$$

\section{Results and discussion}

\subsection{Numerical estimation of material properties}

This section shows computational results of the stiffness and strength of the composite materials. The fiber volume fraction $V_{f}$ was fixed to be 0.56 throughout the different materials. The mechanical properties of composite laminates estimated by the microscale analysis are shown in Table 5 . Here, $E_{\mathrm{QI}}, G_{\mathrm{QI}}$, and $\mathrm{v}_{\mathrm{QI}}$ are the mechanical properties of quasiisotropic laminates and are calculated using the classical laminate theory. Stiffness of the composites using T800S (and equivalently $\mathrm{T} 800 \mathrm{H}$ ) and T1100G higher than that using T700S (and equivalently T300) by $28 \%$ and $40 \%$, respectively.

Table 5 Mechanical properties of composite laminates estimated by micro-scale analysis.

\begin{tabular}{c|c|c|c|c|c|c|c|c|c}
\hline \hline & $E_{L L}^{\mathrm{c}}[\mathrm{GPa}]$ & $E_{T T}^{\mathrm{c}}[\mathrm{GPa}]$ & $G_{L T}^{\mathrm{c}}[\mathrm{GPa}]$ & $G_{T T}^{\mathrm{c}}[\mathrm{GPa}]$ & $v_{L T}^{c}$ & $\boldsymbol{v}_{T T}^{\mathrm{c}}$ & $E_{\mathrm{QI}}[\mathrm{GPa}]$ & $G_{\mathrm{QI}}[\mathrm{GPa}]$ & $v_{\mathrm{QI}}$ \\
\hline Methodology & \multicolumn{7}{|c|}{ Sec. 3.2.2 } \\
\hline T300/\#3900-2B & 127 & 7.6 & 4.0 & 2.6 & 0.26 & 0.54 & 48 & 18.5 & 0.31 \\
T700S/\#3900-2B & 127 & 7.6 & 4.0 & 2.6 & 0.26 & 0.54 & 48 & 18.5 & 0.31 \\
T800H/\#3900-2B & 163 & 7.8 & 4.0 & 2.7 & 0.26 & 0.54 & 60 & 22.9 & 0.31 \\
T800S/\#3900-2B & 163 & 7.8 & 4.0 & 2.7 & 0.26 & 0.54 & 60 & 22.9 & 0.31 \\
T1100G/\#3900-2B & 179 & 8.0 & 4.0 & 2.7 & 0.25 & 0.54 & 66 & 24.9 & 0.32 \\
\hline
\end{tabular}

Strength properties of the composite laminates estimated by the microscale analysis are listed in Table 6 . The strength in the fiber direction is shown in Fig. 6. As the fiber strength increases, the tensile strength in the fiber direction of the composite material significantly increases, while the compressive strength in the fiber direction is approximately constant. It is also noteworthy that, as in Fig. 7, the compressive strength in the direction transverse to the fibers $\left(C_{T T}\right)$ and the shear strength $\left(S_{L T}\right)$ are not significantly affected by fibers since the strength in the direction transverse to the fiber is determined by the elasto-plastic deformation of resin. 
Table 6 Strength properties of composite laminates estimated by micro-scale analysis.

\begin{tabular}{c|c|c|c|c|c}
\hline \hline & $T_{L L}[\mathrm{MPa}]$ & $C_{L L}[\mathrm{MPa}]$ & $T_{T T}[\mathrm{MPa}]$ & $C_{T T}[\mathrm{MPa}]$ & $S_{L T}[\mathrm{MPa}]$ \\
\hline Methodology & Sec. 3.2.3 & Sec. 3.2.4 & \multicolumn{3}{|c}{ Sec. 3.2.5 } \\
\hline T300/\#3900-2B & 1481 & 1625 & 71.78 & 161.25 & 63.40 \\
T700S/\#3900-2B & 2290 & 1625 & 71.78 & 161.25 & 63.40 \\
T800H/\#3900-2B & 2372 & 1600 & 69.96 & 160.64 & 63.40 \\
T800S/\#3900-2B & 2997 & 1600 & 69.96 & 160.64 & 63.40 \\
T1100G/\#3900-2B & 3301 & 1592 & 69.55 & 160.85 & 63.40 \\
\hline
\end{tabular}

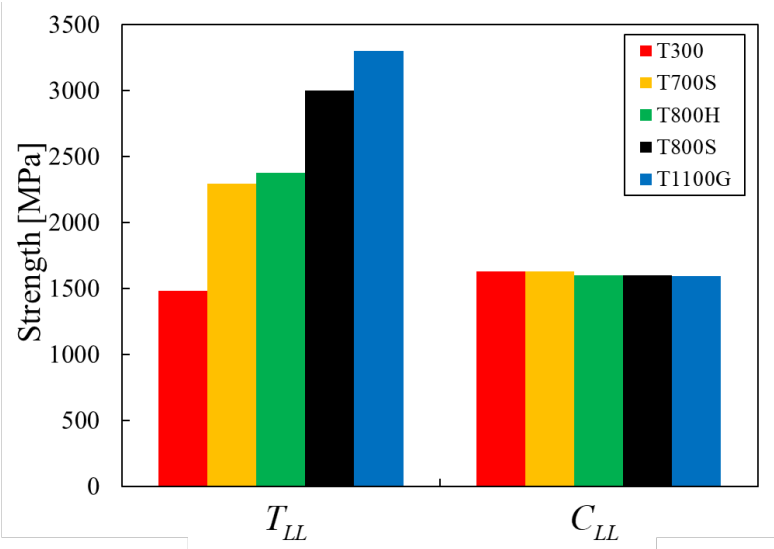

Fig. 6 Longitudinal tensile and compressive strength, estimated using microscale analyses.

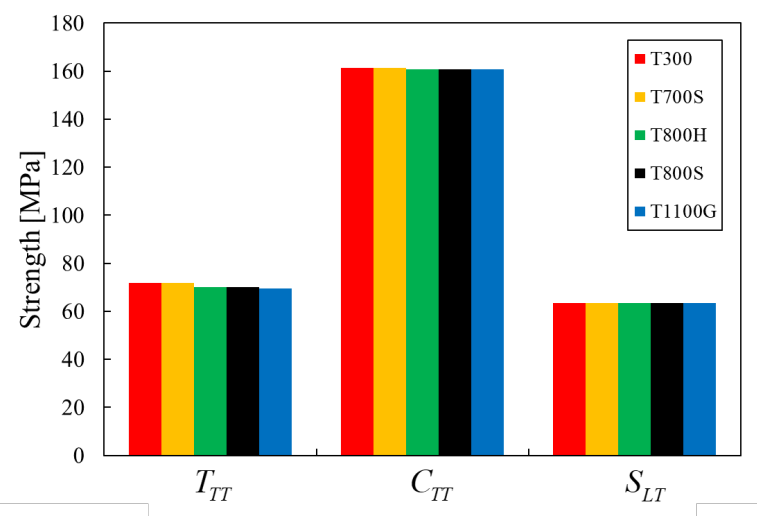

Fig. 7 Transverse tensile and compressive strength and in-plane shear strength, estimated using microscale analyses.

\subsection{Aerodynamic computation and fluid-structure interaction}

Figure 8 shows the surface pressure distributions in the upper and lower sides of the wing. The color contours represent the pressure coefficient, $C p$, at a freestream Mach number of 0.84 . The $C p$ distribution is smooth and broadly positive on the lower surface. The pressure is locally maximized along the leading-edge line (from orange to red contour region in Fig. 8) due to a stagnation of the flow, and a higher-pressure region appears again in the vicinity of the trailing edge because of the positive camber of the RAE2822 airfoil. The upper surface is, on the other hand, broadly covered by negative $C p$ values associated with a strong discontinuity near the trailing edge. The discontinuity of the $C p$ corresponds to a shock wave, which is typically observed in transonic aircraft configurations (Martins, et al.,2001, Kenway and Martins, 2014), and the strong suction peak appears in the fore side of the shock wave. The overall aerodynamic force acting on each wing section is vertically positive, and thus the lift force is generated in the present wing configuration. Note that the location of a shock wave strongly depends on the airfoil shape and freestream conditions such as Mach number and angle of attack as well as computational conditions. The right figures of Fig. 8 show the $C p$ distributions using different grids, where the number of grid points is $87 \times 38 \times 50$ and $297 \times 143 \times 100$ for the coarse and fine grid, respectively. The $C p$ distributions of the coarse and fine grids are not significantly different from that of the medium grid 
(the left figure in Fig. 8), and thus the aerodynamic load considered herein is sufficiently converged in terms of a grid density study.

On the load transfer from the aerodynamic grid to the structure grid, the force was exchanged between the nearest four grid points. Therefore, the force acting on the upper and lower panels of the wingbox structure is not always vertically positive, e.g., the mid chord region of the lower wing surface where the $C p$ becomes negative. Furthermore, in this study, the aerodynamic and structural analyses are one-way coupled in the sense of FSI, where the deformation of the wingbox structure is not considered in the aerodynamic simulation (Feripa et al., 2001). It is not necessary to consider a two-way coupling unless strong interaction exists between the structural deformation and associated aerodynamic loads in terms of FSI viewpoint (Piperno et al., 1995, Feripa et al., 2001). Indeed, there was a report which shows that the deformation of the wingbox structure does not significantly affect the aerodynamic characteristics in the transonic flight condition (Martins et al., 2001). However, the aforementioned references thoroughly focused on metal wings which do not significantly deform due to high stiffness of the wing structure. The present study focuses on CFRP wings which can largely deform, and thus the effect of wing deformation on the aerodynamic load may not be negligible. The effects of wing deformation on the aerodynamic load has been reported earlier in Obayashi 1995 and more recently pointed out in the context of the wing shape optimization for CFRP wings (Kenway and Martins, 2014, Kennedy and Martins, 2012). The effects of wing deformation on the aerodynamic load need to be considered as a next step by performing iterative (two-way coupling) calculations of fluid and structure analyses to obtain a converged aerostructural solution as a static aeroelastic problem including structural sizing process. This is, however, not often straightforward due to the nature of nonlinearlity by combining aeroelastic problem with structural sizing in the iterative process.
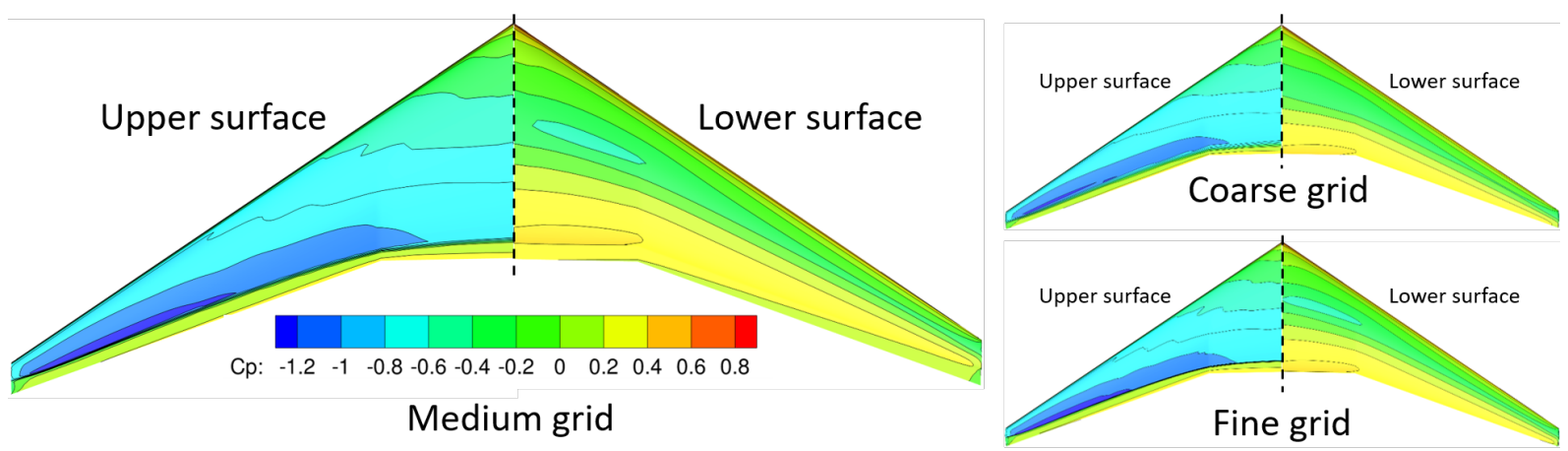

Fig. 8 Surface pressure distribution of the wing.

\subsection{Effects of fiber properties on structural sizing for a CFRP wing design}

Figure 9 shows the structural weights of the entire wing structures using T300, T700S, T800H, T800S, and T1100G. Figure 9 a) and b) show the results using the Christensen's criterion and the maximum stress criterion in Eq. (6) for the fiber-dominant failure criterion. It can be seen that the weights using the Christensen's criterion are slightly heavier than those using the maximum stress criterion. The details of the difference between those criteria will be discussed in the next paragraph. Meanwhile, in each criterion, the wings with stiffer fiber becomes lighter in terms of the entire wing structures. It is consistent with the well-known fact that the structural weight becomes lighter when the stiffness and strength of material are higher. On the other hand, the order of the weight (see the dark blue and light blue bars in Fig. 9 a) and b)) is opposite between the upper and lower panels: the weight of the lower panel increases as the stiffness of the fiber increases (i.e., from T700S to T1100G), while the weight of the upper panel decreases as the stiffness of the fiber increases. This characteristic behavior will be also discussed in the following two paragraphs. 


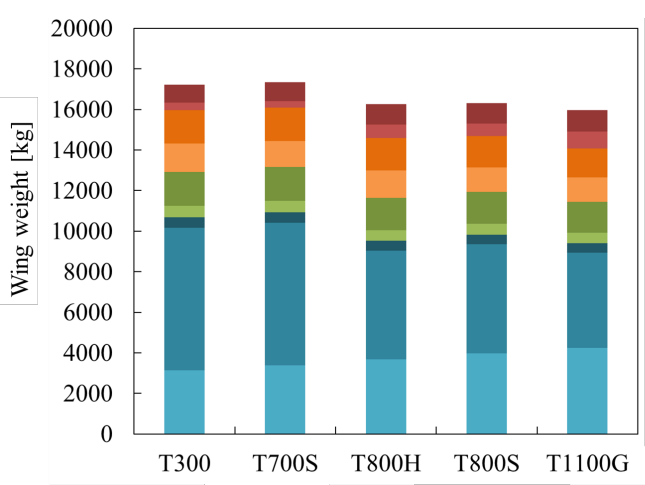

a) Christensen's criterion

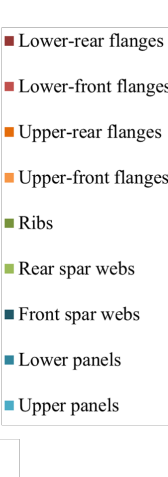

Fig. 9 Wing weights using T300, T700S, T800H, T800S, and T1100G.

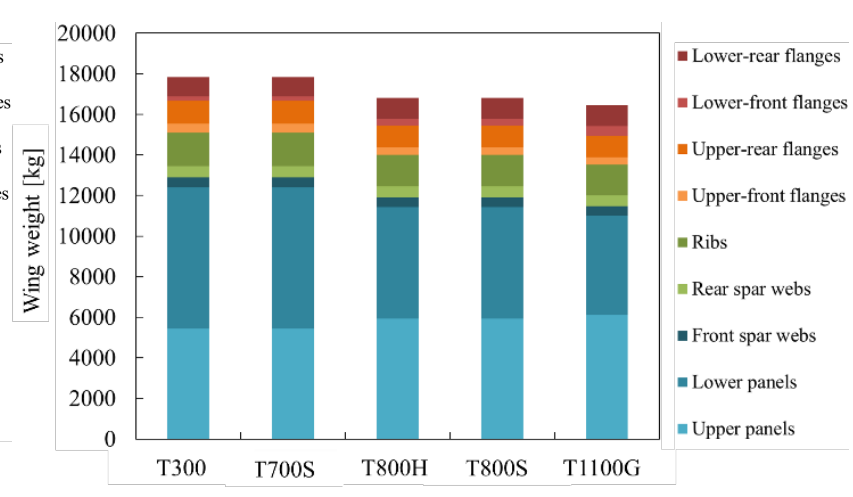

b) Maximum stress criterion

Figure 10 shows the thickness distributions of the upper panels with different fibers. The horizontal axis denotes the index of rib stations which ranges between 0 (at the wing root) and 35 (at the wing tip). Note that the thickness of the panel is uniform in the chordwise direction, and thus the plot of the upper panel thickness can be uniquely defined for each fiber. As suggested from the difference of upper panel weights between two criteria in Fig. 9, the overall thickness of the upper panel with the Christensen's criterion is lower than that with the maximum stress criterion in Fig. 10. Further discussion will be performed along with the failure modes on upper panels. Figures 11-16 show the failure modes of upper panels of T700S, T800S, and T1100G, where the contour color represents the M.S. between 0 and 1 . The other cases, $\mathrm{T} 300$ and $\mathrm{T} 800 \mathrm{H}$, are omitted for brevity, which are not significantly different from Figs. 11-16. The green region indicates that the M.S. is approximately 0.5 , where the corresponding failure mode is likely dominant. From Figs. $11 \mathrm{~b}$ )$16 \mathrm{~b}$ ), it is suggested that in the most parts of the upper panels (i.e., from wing root to approximately the mid span of the wing), the structural sizing is based on the compressive failure in the longitudinal direction. Regardless of the failure criteria, the thickness tends to increase when the stiffness of the fibers increases. This trend is explained by the compressive strength $C_{\mathrm{QI}}$ of the quasi-isotropic laminates as follows:

$$
C_{\mathrm{QI}}=\frac{C_{L L}}{E_{L L}^{\mathrm{c}}} E_{\mathrm{QI}}
$$

where the strain in each layer is assumed to be the same, which is determined by the 0 -degree layer. Let us focus on the ratio of the stiffness of the unidirectional laminate $\left(E_{L L}\right)$ to that of the quasi-isotropic laminate $\left(E_{\mathrm{QI}}\right)$ in Eq. (17). Figure 17 shows the value of $E_{\mathrm{QI}} / E^{c}{ }_{L L}$ with respect to the fiber stiffness $E_{L L}^{\mathrm{f}}$. It is interesting that $E_{\mathrm{QI}} / E^{c}{ }_{L L}$ decreases as the stiffness of the fiber increases. Hence, as shown in Fig. 18, the compressive strength of the quasi-isotropic laminates $C_{\mathrm{QI}}$ decreases as the stiffness of the fiber increases even if the compressive strength $C_{L L}$ is approximately the same among the fibers. Accordingly, the thickness of T1100G becomes the largest among five fibers with both criteria in Eq. (6). Meanwhile, in Fig. 10, the difference in thickness between fibers is larger with the Christensen's criterion than that with the maximum stress criterion. Furthermore, particularly in the Christensen's case, the thickness of T300 (or T800H) is different from that of T700S (or T800S) although the stiffness of fibers is the same. This is explained by the fact that when the Christensen's criterion is used along with the non-zero M.S., the allowable compressive strength corresponding to $M . S .=0.5$ varies greatly depending on the tensile strength. This is due to the nature of the Christensen's criterion for the fiber-dominant failure mode, which is explained in the equation below:

$$
\sigma_{L L}=\frac{-\left(C_{L L}-T_{L L}\right) \pm \sqrt{\left(C_{L L}-T_{L L}\right)^{2}+4 \frac{T_{L L} C_{L L}}{1+M \cdot S .}}}{2}
$$

Equation (18) is given by Eq. (6) with Eq. (15) for each sizing iteration step and represents the allowable compressive strength assuming that $\sigma_{T T}=0$. If $M . S=0$, Eq. (18) corresponds to $C_{L L}$. However, if $M . S$ is non-zero, the tensile strength is included in the right-hand side of Eq. (18). This yields the difference of panel thickness between T300 (or T800H) and T700S (or T800S) although the fiber stiffness is the same. The use of Eq. (18) also leads to the large difference of allowable compressive stress at M.S. $=0.5$ among five fibers, and thus the difference of panel thickness is larger with the 
Christensen's criterion in Fig. 10 a). On the other hand, such a large difference is not observed when the maximum stress criterion is adopted. As such, the use of the fiber-dominant Christensen's criterion along with the non-zero M.S. in the form of Eqs. (6) and (15) would yield unreasonable results, which should be carefully treated in the context of structural design. Meanwhile, the panel thickness in the vicinity of the wing tip is in the order of fiber stiffness in Fig. 10. This is consistent with the fact that the local buckling is dominant in Figs. 11-16, where the fiber microbuckling does not affect the structural sizing.

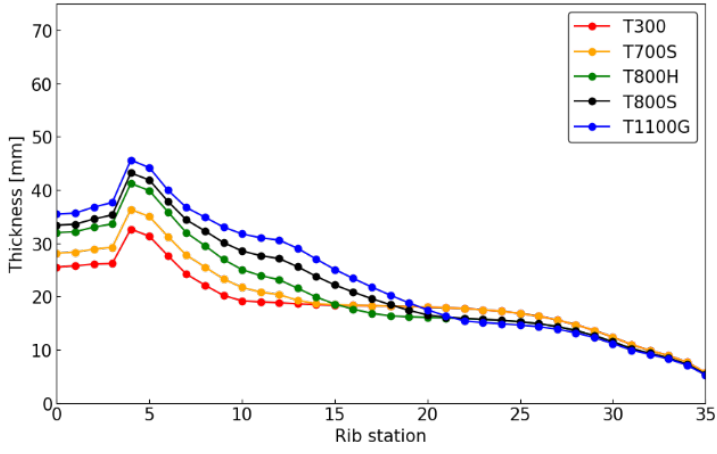

a) Christensen's criterion

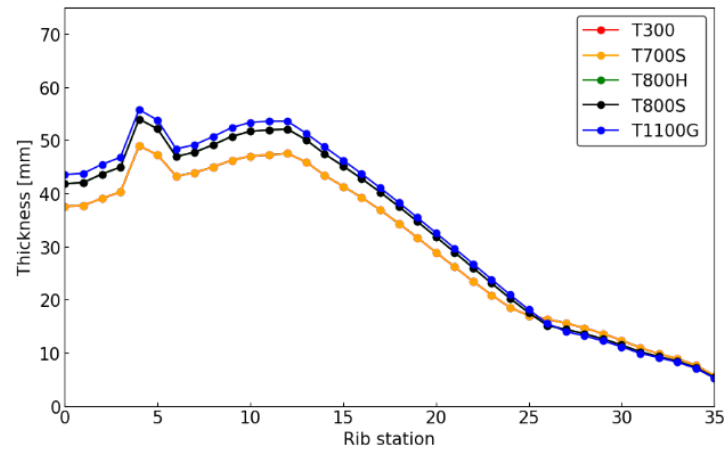

b) Maximum stress criterion

Fig. 10 Distributions of shell element thickness of upper panels. 


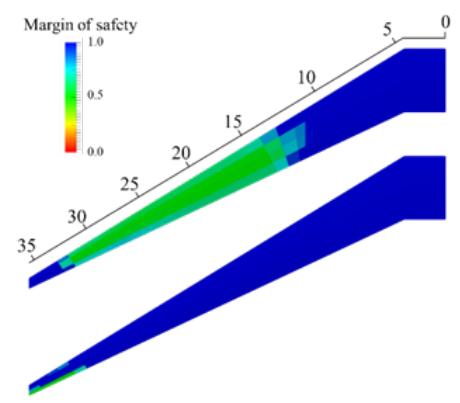

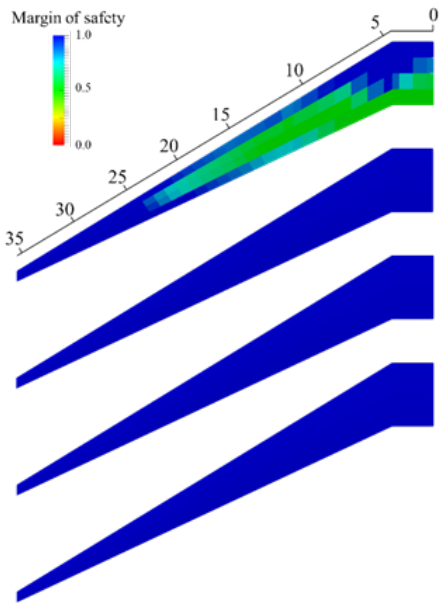

b) Fiber dominant failure (0/45/-45/90 degree layer)

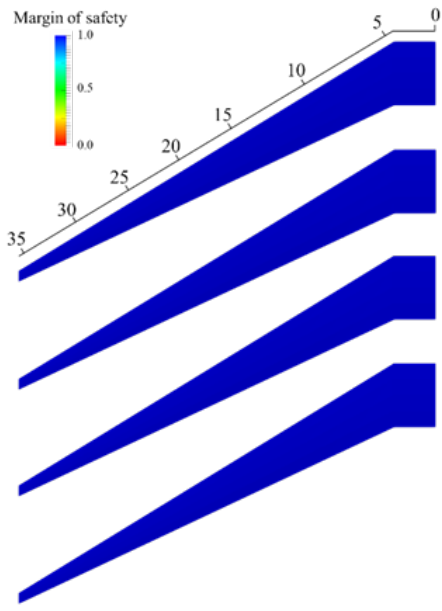

c) Matrix dominant failure (0/45/-45/90 degree layer)

Fig. 11 The distribution of M.S. on upper skin for each failure modes using T700S with Christensen's criterion.

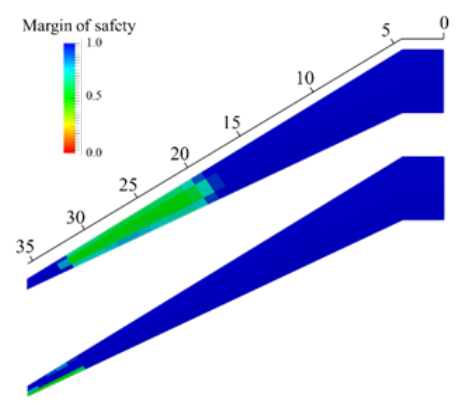

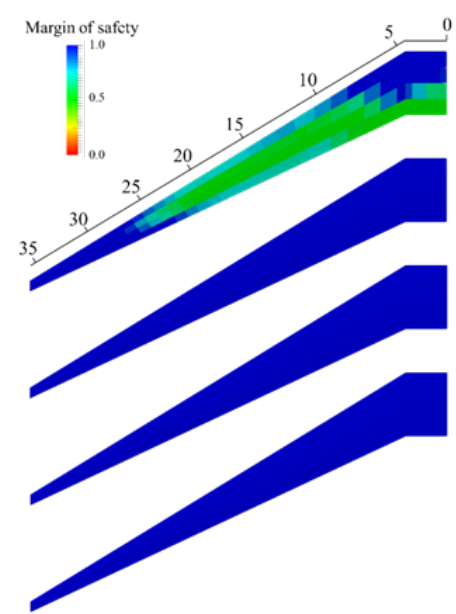

b) Fiber dominant failure (0/45/-45/90 degree layer)

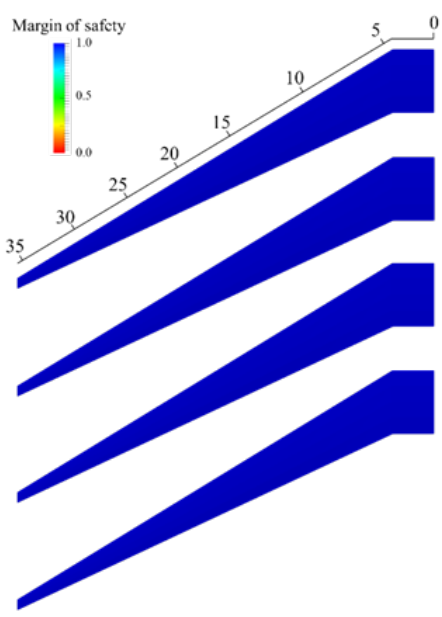

c) Matrix dominant failure (0/45/-45/90 degree layer)

Fig. 12 The distribution of M.S. on upper skin for each failure modes using T800S with Christensen's criterion.

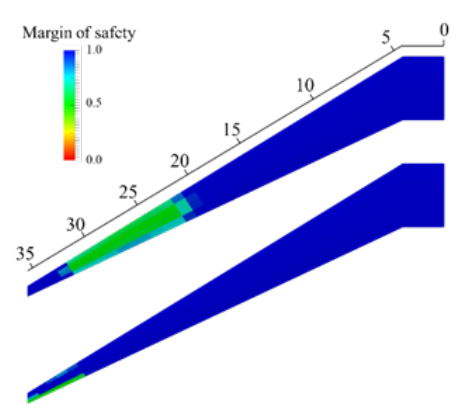

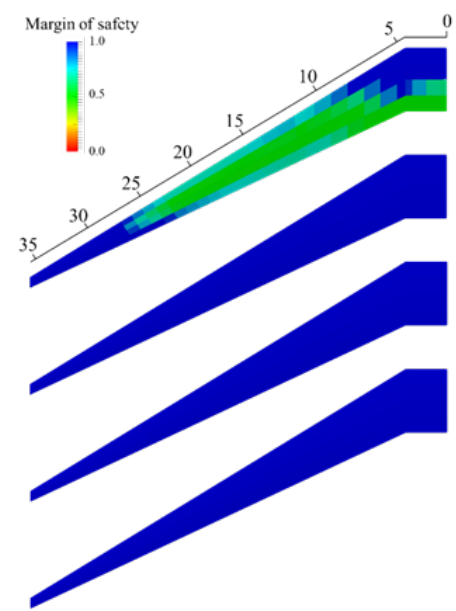

b) Fiber dominant failure (0/45/-45/90 degree layer)

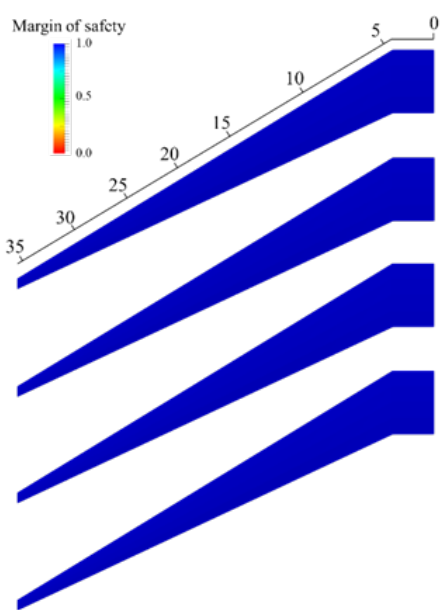

c) Matrix dominant failure (0/45/-45/90 degree layer) (compression, shear)

Fig. 13 The distribution of M.S. on upper skin for each failure modes using T1100G with Christensen's criterion. 


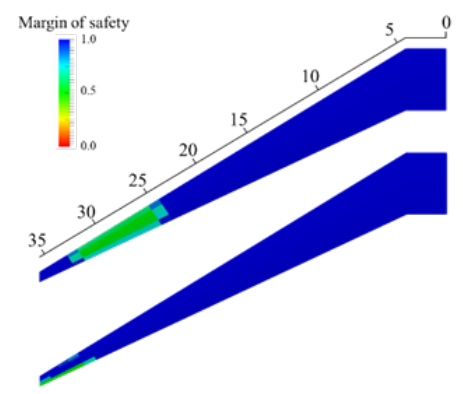

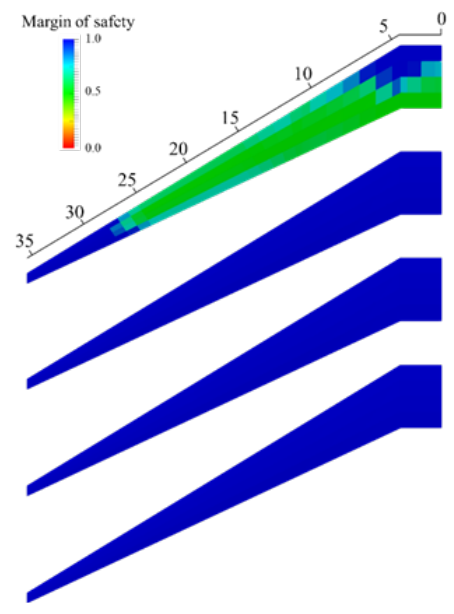

b) Fiber dominant failure (0/45/-45/90 degree layer)

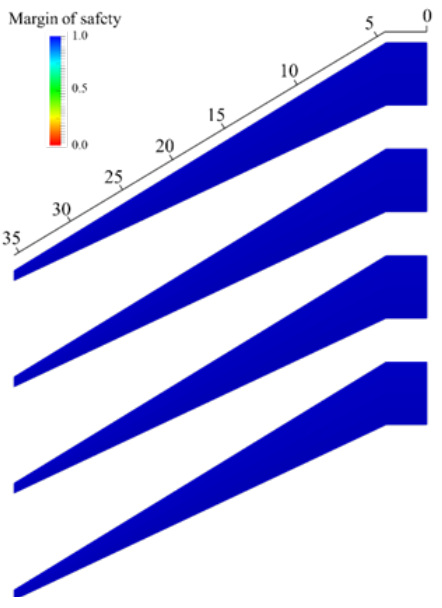

c) Matrix dominant failure (0/45/-45/90 degree layer)

Fig. 14 The distribution of M.S. on upper skin for each failure modes using T700S with maximum stress criterion.

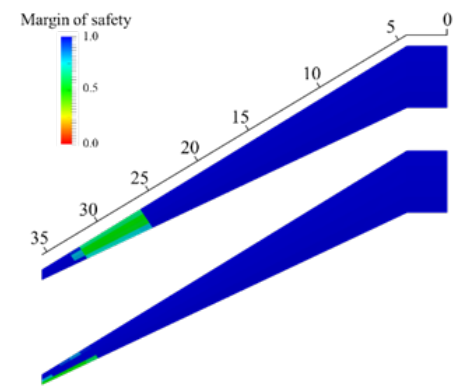

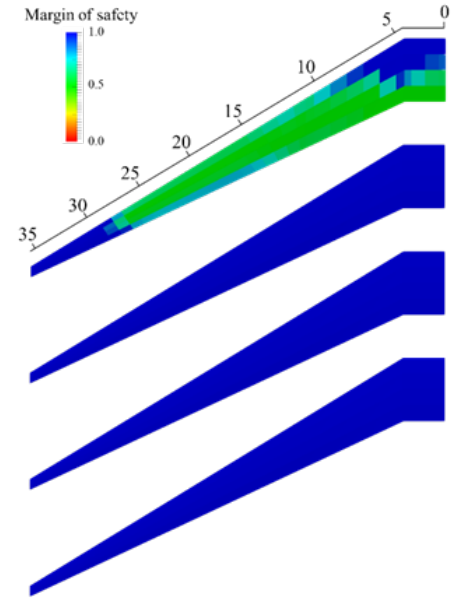

b) Fiber dominant failure (0/45/-45/90 degree layer)

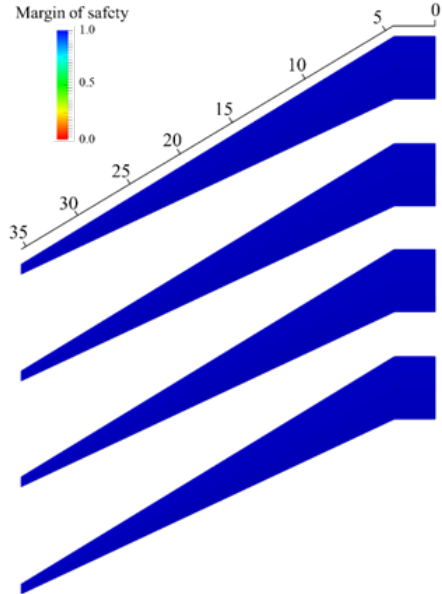

c) Matrix dominant failure (0/45/-45/90 degree layer)

Fig. 15 The distribution of M.S. on upper skin for each failure modes using T800S with maximum stress criterion.

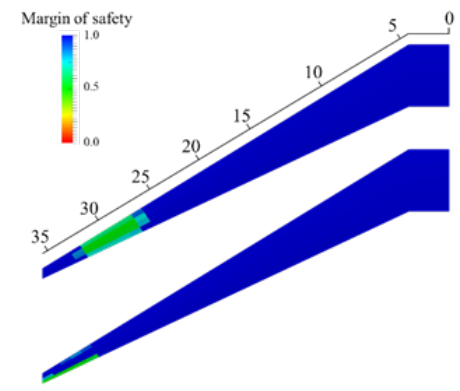

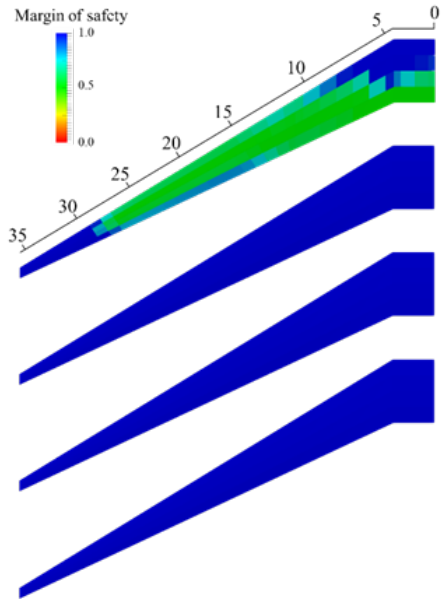

b) Fiber dominant failure (0/45/-45/90 degree layer)

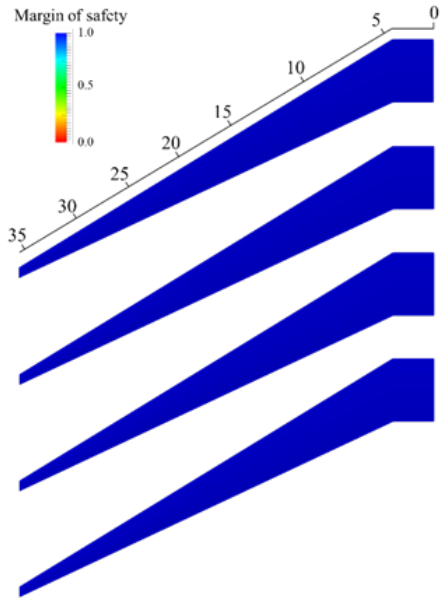

c) Matrix dominant failure (0/45/-45/90 degree layer)

Fig. 16 The distribution of M.S. on upper skin for each failure modes using T1100G with maximum stress criterion. 


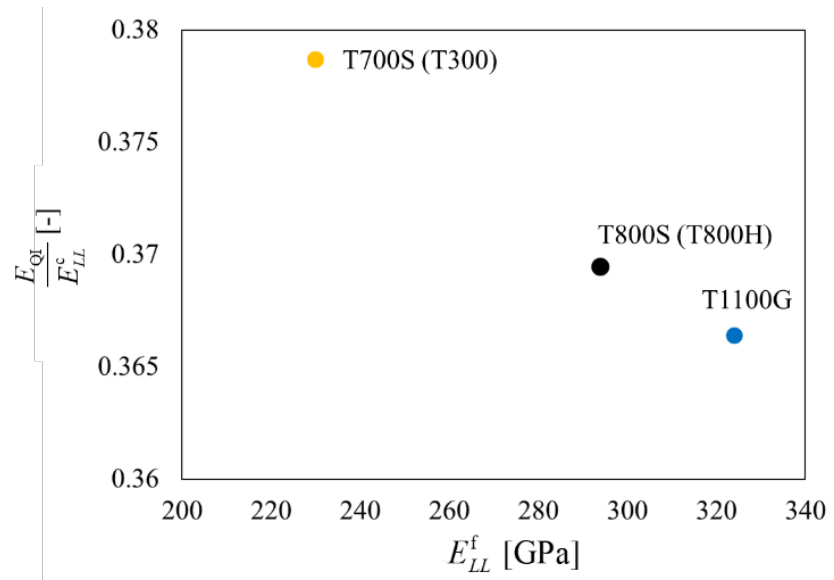

Fig. 17 Ratio of the stiffness of unidirectional laminate to the stiffness of the quasi-isotropic laminate

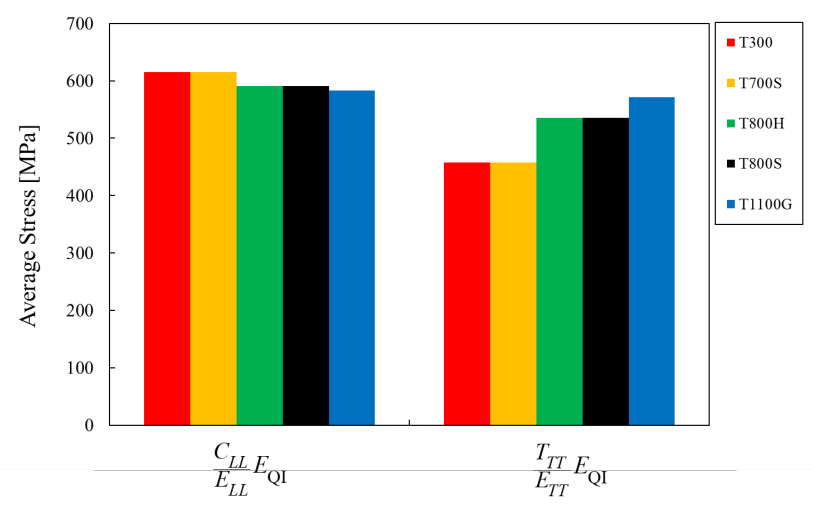

Fig. 18 Averaged stress of quasi-isotropic laminates at critical condition.

Figure 19 shows the thickness distributions of the lower panels. It is found that the sizing was conducted in most parts of the lower panels based on the tensile strength in the transverse direction as shown in Figs. 20-25. Regardless of the failure criteria, the thickness tends to decrease significantly when the stiffness of the fibers is increased. In the case of tensile condition, it is observed that the tensile stress of the quasi-isotropic laminates with T1100G shows the highest value in Fig. 18. Therefore, the wing with T1100G shows the lowest thickness in overall lower panels.

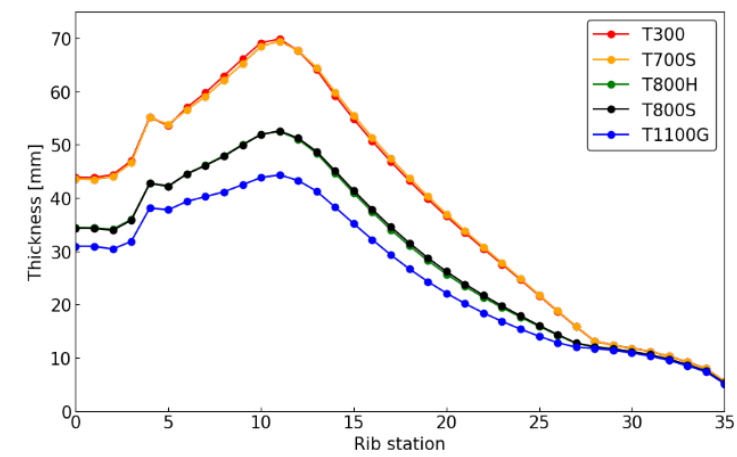

a) Christensen's criterion

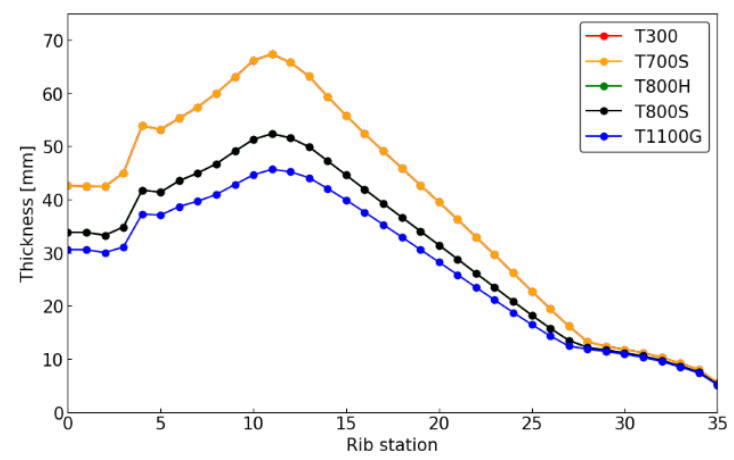

b) Maximum stress criterion

Fig. 19 Distributions of shell element thickness of the lower panels. 


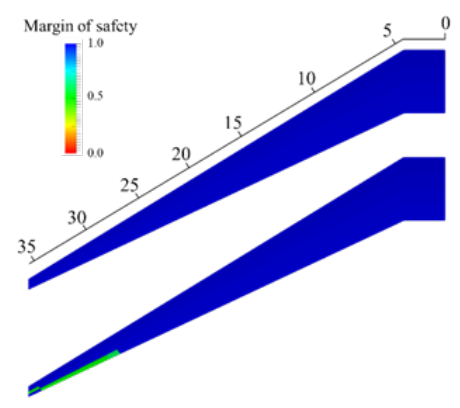

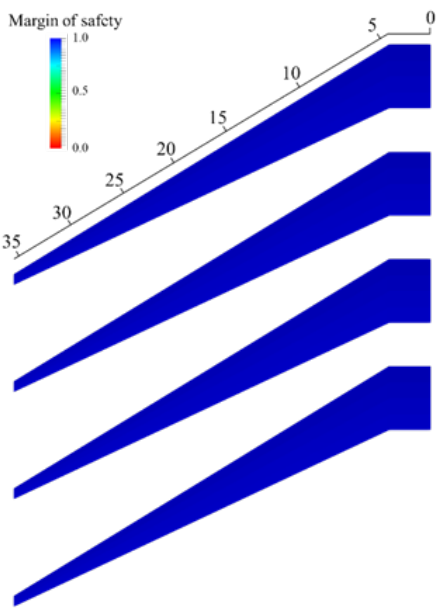

b) Fiber dominant failure (0/45/-45/90 degree layer)

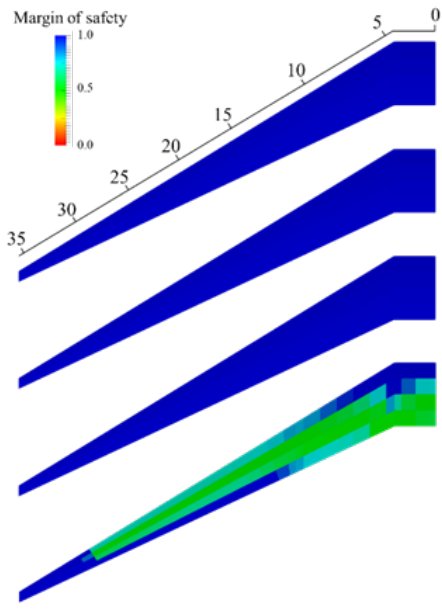

c) Matrix dominant failure (0/45/-45/90 degree layer)

(compression, shear)

Fig. 20 The distribution of M.S. on lower skin for each failure modes using T700S with Christensen's criterion.

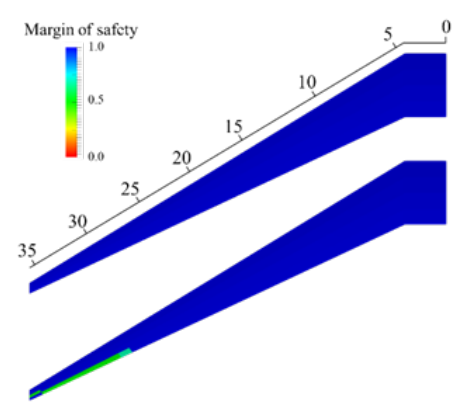

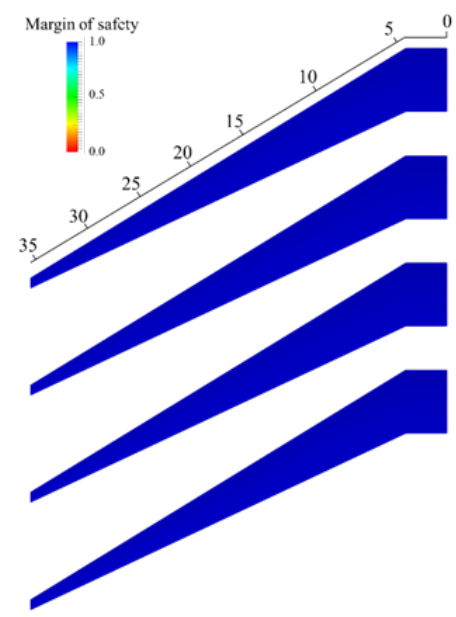

b) Fiber dominant failure (0/45/-45/90 degree layer)

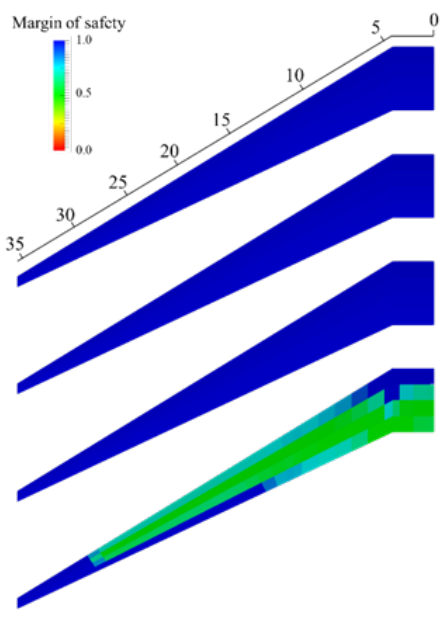

c) Matrix dominant failure (0/45/-45/90 degree layer) (compression, shear)

Fig. 21 The distribution of M.S. on lower skin for each failure modes using T800S with Christensen's criterion.

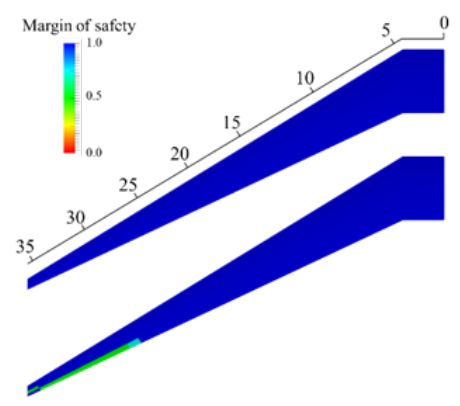

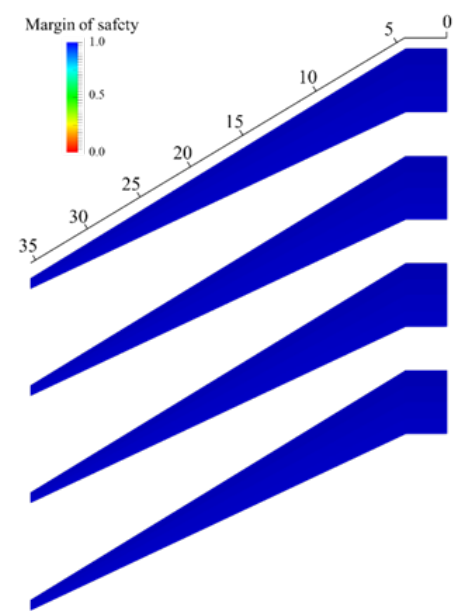

b) Fiber dominant failure (0/45/-45/90 degree layer)

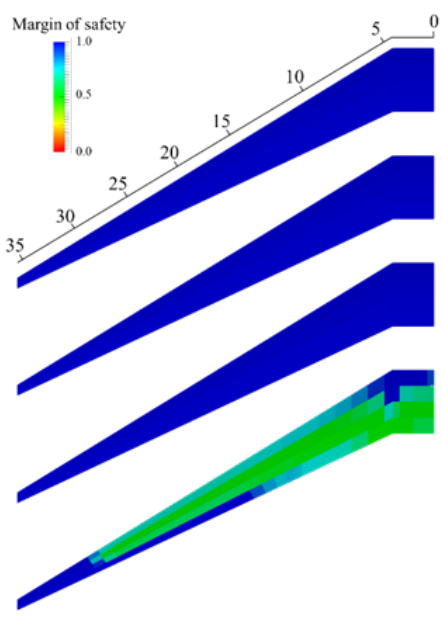

c) Matrix dominant failure (0/45/-45/90 degree layer) (compression, shear)

Fig.22 The distribution of M.S. on lower skin for each failure modes using T1100G with Christensen's criterion. 


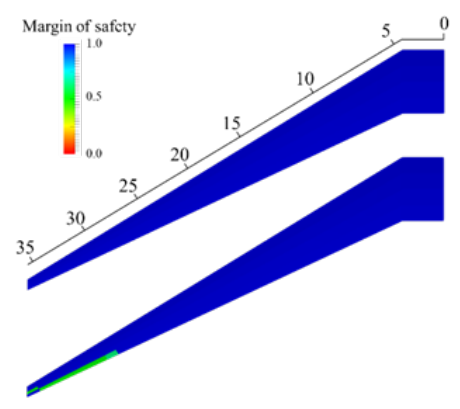

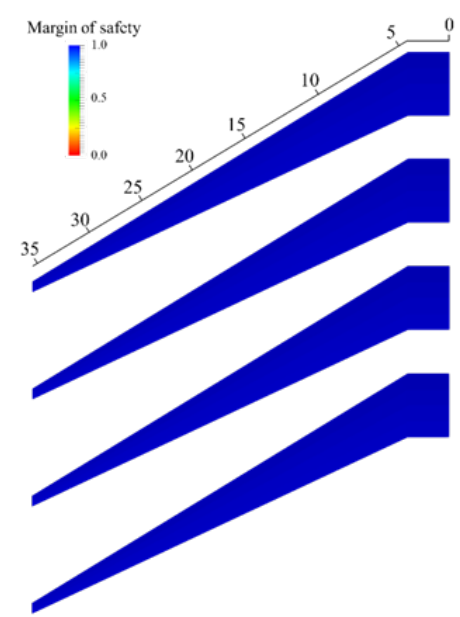

b) Fiber dominant failure (0/45/-45/90 degree layer)

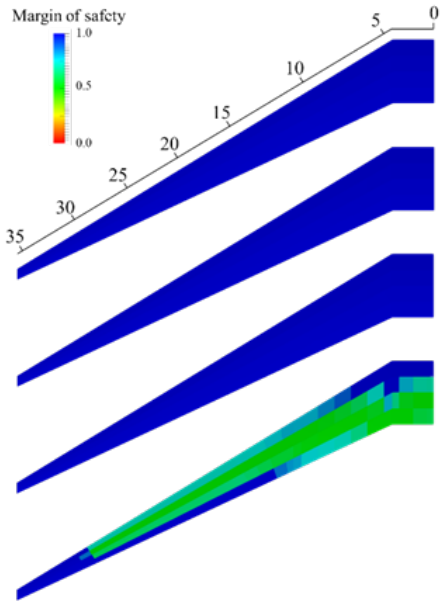

c) Matrix dominant failure (0/45/-45/90 degree layer) (compression, shear)

Fig. 23 The distribution of M.S. on lower skin for each failure modes using T700S with maximum stress criterion.

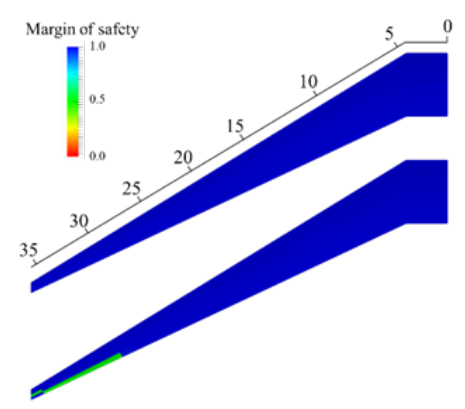

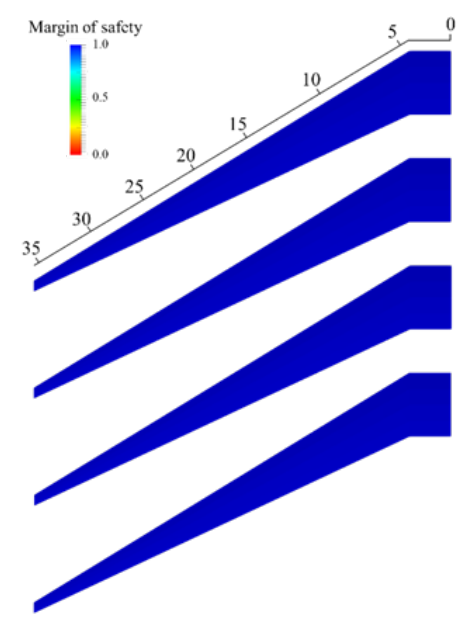

b) Fiber dominant failure (0/45/-45/90 degree layer)

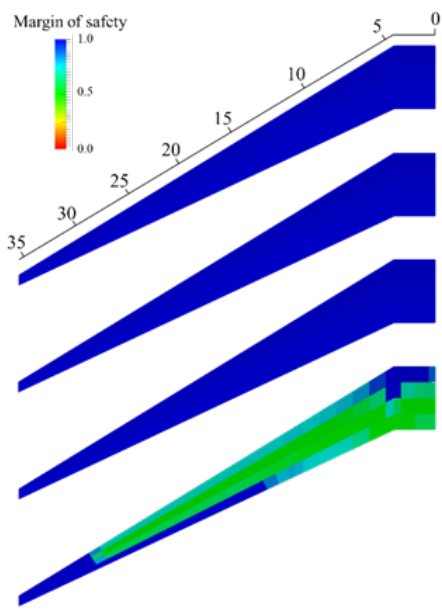

c) Matrix dominant failure (0/45/-45/90 degree layer)

Fig. 24 The distribution of M.S. on lower skin for each failure modes using T800S with maximum stress criterion.

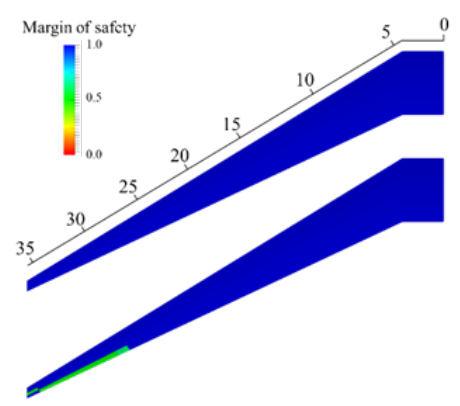

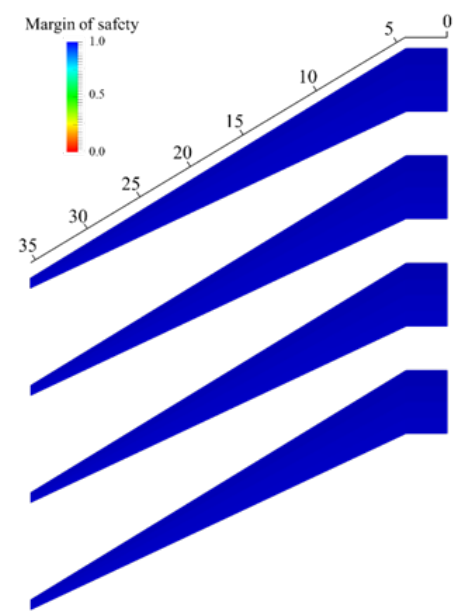

b) Fiber dominant failure (0/45/-45/90 degree layer)

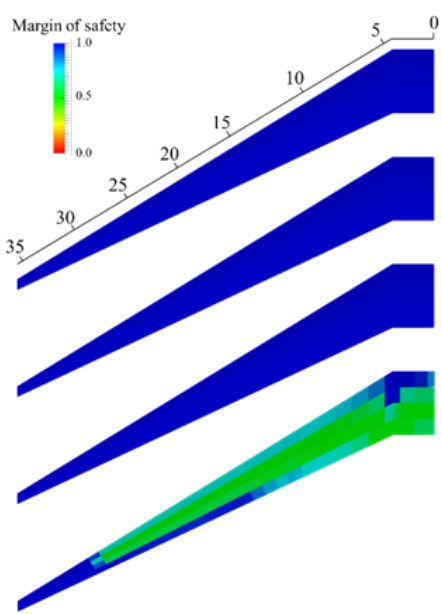

c) Matrix dominant failure (0/45/-45/90 degree layer)

Fig. 25 The distribution of M.S. on lower skin for each failure modes using T1100G with maximum stress criterion. 


\section{Conclusion}

In this paper, we investigated the difference of wings' structural weights using CFRPs with five fibers: T300, T700S, $\mathrm{T} 800 \mathrm{H}, \mathrm{T} 800 \mathrm{~S}$ and T1100G. To perform the investigation, a multiscale framework for designing composite aircraft wings has been developed. In the microscale analysis, four methods were utilized sequentially to consider the different fracture mechanisms of CFRP. First, PUC analysis based on the FEA was performed to obtain unidirectional laminates with homogenized stiffness. To estimate anisotropic strength, SEM, the Sun-Jun model, and PUC analysis were used to measure resin damage. In the macro-scale analysis, one-way aeroelastic analysis was performed, where the compressible Euler solver and the static solver were used. The aerodynamic load corresponding to the calculated pressure distribution around the wing was transferred to a structural model by applying the CVT method, and structural analysis was performed using FEA. Finally, the stress state obtained from the structural analysis was used to design the wingbox under the constraints of Christensen's failure criteria and maximum stress criterion as well as the local buckling criterion. The mechanical properties (stiffness and strength) of composite laminates used in the structural analysis and failure criteria are the values estimated by microscale analysis.

The microscale analysis revealed the effects of different properties of the fiber and resin on the mechanical properties of the composite material. In terms of the weight of entire wing structures, the wings with T1100G resulted in the lightest structure. It was also observed that a trend in the weight variance with respect to the fibers is opposite between the upper and lower panels: the weight of the upper panel is increased as the stiffness of the fiber increases (i.e., from T300 to T1100G) while the weight of the lower panel is decreased as the stiffness of the fiber increases. In the most part of the upper panels, T1100G showed the largest thickness in spite of the highest stiffness in the five fibers. This was explained by the fact that the compressive strength of the quasi-isotropic laminates decreases as the stiffness of the fiber increases even if the compressive strength is approximately the same among the fibers. Furthermore, it was also indicated that the use of the Christensen's criteria for the fiber-dominant failure along with the non-zero M.S. may lead to unreasonable results as the allowable compressive stress would be largely affected not only by the compressive strength but also by the tensile strength in the longitudinal direction. Meanwhile, the upper-panel thickness around the wing tip was in the order of fiber stiffness, where the local skin buckling was dominant. For the lower panel, the thickness decreased significantly as the stiffness of the fibers increased, where the tensile strength in the transverse direction. Consequently, the contribution of the lower panel to the entire wing weight is larger than that of the upper panel, and thus the entire wing weight becomes the lightest with $\mathrm{T} 1100 \mathrm{G}$ of the highest stiffness among the five fibers. Finally, it should be noted that this study presented the results of the multiscale analysis applied to limited wing configuration, resin, and stacking sequence. The above limitations of this work can be further investigated in the future study. 


\section{Acknowledgements}

The authors wish to acknowledge Dr. Toshio Nagashima, professor of Sophia University, for his significant help in performing the structural analysis. This work was supported by Council for Science, Technology and Innovation (CSTI), Cross-ministerial Strategic Innovation Promotion Program (SIP), "Materials Integration" for revolutionary design system of structural materials (Funding agency: JST).

\section{References}

Asp, L., E., Berglund, L., A., and Talreja, R., A criterion for crack initiation in glassy polymers subjected to a compositelike stress state, Composites Science and Technology, Vol. 56, (1996), pp. 1291-1301.

Baker, A. A., and Scott, M., L., Composite Materials for Aircraft Structures (2004), p. 2, 87, AIAA Education Series, American Institute of Aeronautics and Astronautics.

Bathe, K., J., Finite element procedures (2006), Klaus-Jurgen Bathe.

Bram, V., T., Towards the Ultimate Conservative Difference Scheme. V. A Second-Order Sequel to Godounov's Method, Journal of Computational Physics, Vol. 32, (1979), pp. 101-136.

Christensen, R., M., Stress based yield/failure criteria for fiber composites, International Journal of Solids and Structures, Vol. 34, (1997), pp. 529-543.

Du, X., Amrit, A., Thelen, A. Leifsson, L., Aerodynamic design of a rectangular wing in subsonic inviscid flow by surrogate-based optimization, Proceedings of the 35th AIAA Applied Aerodynamics Conference (2017).

Dvorkin, E., N., Bathe, K., J., A continuum mechanics based four-node shell element for general non-linear analysis, Engineering Computations, Vol. 1, No. 1 (1984), pp. 77-88.

Felippa, C.A., Park, K.C., Farhat, C., Partitioned analysis of coupled mechanical systems, Computer Methods in Applied Mechanics and Engineering, Vol. 190 (2001), No. 24, pp. 3247-3270.

Goura, G., S., L, Time Marching Analysis of Flutter Using Computational Fluid Dynamics, Ph. D. thesis, University of Glasgow, (2001).

Gurson, A., L., Continuum theory of ductile rupture by void nucleation and growth: Part I-Yield criteria and flow rules for porous ductile media, Journal of engineering materials and technology, Vol. 99, (1977), pp. 2- 15.

Kelly, A., and Tyson, W., Tensile properties of fibre-reinforced metals: Copper/tungsten and copper/molybdenum, Journal of the Mechanics and Physics of Solids, Vol. 13, (1965), pp. 329-350.

Kennedy, G., Martins, J., A Comparison of Metallic and Composite Aircraft Wings Using Aerostructural Design Optimization, Proceedings of the 12th AIAA Aviation Technology, Integration, and Operations (ATIO) Conference and 14th AIAA/ISSMO Multidisciplinary Analysis and Optimization Conference (2012).

Kennedy, G., Kenway, G. W., Martins, J. R. R. A., High Aspect Ratio Wing Design: Optimal Aerostructural Tradeoffs for the Next Generation of Materials, Proceedings of the 52nd Aerospace Sciences Meeting (2014).

Kenway, G. W., and Martins, J. R. R. A., Multi-Point High-Fidelity Aerostructural Optimization of a Transport Aircraft Configuration, Journal of Aircraft, Vol. 51, (2014), pp. 144-160.

Kobayashi, S., Tomii, D., and Shizawa, K., A modelling and simulation on failure prediction of ductile polymer based on craze evolution and annihilation, Transactions of the Japan Society of Mechanical Engineers (in Japanese), Vol. 70, (2004), DOI: 10.1299/kikaia.70.810

Lansing, W., Dwyer, W., Emerton, R., and Ranalli, E., Application of Fully Stressed Design Procedures to Wing and Empennage Structures, Journal of Aircraft, Vol. 8, (1971), pp. 683-688.

LLorca, J., Gonzalez, C., Molina-Aldareguia, J. M., Segurado, J., Seltzer, R., Sket, F., Rodriguez, M., Sadaba, S., Munoz, R., and Canal, L.P., Multiscale modeling of composite materials: A roadmap towards virtual testing, Advanced Materials, Vol. 23, (2011), pp. 5130-5147.

Loidl, D., Paris, O., Rennhofer, H., Muller, M., and Peterlik, H., Skin-core structure and bimodal Weibull distribution of the strength of carbon fibers, Carbon, Vol. 45, (2007), pp. 2801-2805.

Lutz, T., Wagner, S., Numerical shape optimization of subsonic airfoil sections, Proceedings of the European Congress on Computational Methods in Applied Sciences and Engineering (ECCOMAS) (2000), pp. 11-14.

Lyu, Z., Kenway, G. K., and Martins, J.R.R.A., AerodynamicShape Optimization Studies on the Common Research Model Wing Benchmark, AIAA Journal, Vol. 53, (2015), pp. 968-985. 
Matsuda, T., Ohno, N., Tanaka, H., and Shimizu, T., Homogenized in-plane elasticviscoplastic behavior of long fiberreinforced laminates, JSME International Journal, Series A: Solid Mechanics and Material Engineering, Vol. 45, (2002), pp. 528-544.

Martins, J.R.R.A., Alonso,J.J., and Reuther,J.J., Aero-StructuralWing Design Optimization Using High-Fidelity Sensitivity Analysis, Proceedings of the CEAS Conference on Multidisciplinary Aircraft Design and Optimization, edited by Hölinger, H., June 2001, pp. 211-226.

Okabe, T., Sekine, H., Ishii, K., and Takeda, N., Numerical method for failure simulation of unidirectional fiberreinforced composites with spring element model, Composites Science and Technology, Vol. 65, (2005), pp. 921933.

Okabe, T., Kumagai, Y., Higuchi, R., and Nishikawa, M., Onset of Matrix Cracking in Fiber Reinforced Polymer Composites: A Historical Review and a Comparison Between Periodic Unit Cell Analysis and Analytic Failure Criteria, Advanced Structured Materials 64, Altenbach, H. (eds), Springer International Publishing Switzerland, (2015), pp. 299-317.

Obayashi, S., and Guruswamy, G.P., Convergence acceleration of a Navier-Stokes solver for efficient static aeroelastic computations, AIAA journal (1995), Vol.33(6), pp.1134-1141.

Obayashi, S. Jeong, S., Chiba, K., Morino, H., Multi-Objective Design Exploration and Its Application to Regional-Jet Wing Design, Transactions of the Japan Society for Aeronautical and Space Sciences, Vol. 50, (2007), pp. 1-8.

Piperno, S., Farhat, C., and Larrouturou, B., Partitioned procedures for the transient solution of coupled aeroelastic problems Part I: Model problem, theory and two-dimensional application, Computer Methods in Applied Mechanics and Engineering, Vol. 124, (1995) Issues 1-2, pp. 79-112.

Rosen, B., W., Mechanics of composite strengthening, Composite Science and Technology, Vol. 52, (1994), pp. $577-587$.

Sun, C., T., Jun, A., W., Compressive strength of unidirectional fiber composites with matrix non-linearity, Composites Science and Technology, Vol. 52, (1994), pp. 577-587.

Toray Carbon Fibers America, Inc., available from $<$ https://toraycma.app.box.com/s/2uja1lo5to75or5matg9ln0sdyq3ml79>, (accessed on 25, June 2020)

Toray Carbon Fibers America, Inc., available from $<$ https://toraycma.app.box.com/s/bwbxiqtt9e8g33uowkkfurhw303496s0>, (accessed on 25, June 2020)

Yamamoto, G., Onodera, M., Koizumi, K., Watanabe, J., Okuda, H., Tanaka, F., Okabe, T., Considering the stress concentration of fiber surfaces in the prediction of the tensile strength of unidirectional carbon fiber-reinforced plastic composites, Composites Part A, Vol. 121, (2019), pp. 499-509.

Yoon, S., and Jameson, A., Lower-upper symmetric-gauss-seidel method for the euler and navier-stokes equations, AIAA Journal, Vol. 26, (1988), pp. 1025-1026.

Yoshioka, K., Kumagai, Y., Higuchi, R., Lee, D., and Okabe, T., Multiscale modeling of failure strain in off-axis tensile testing of UD-CFRP, Materials system, Vol. 34, (2016), pp. 7-13 (in Japanese).

Watanabe, J., Tanaka, F., Okuda, H., and Okabe, T., Tensile strength distribution of carbon fibers at short gauge lengths, Advanced Composite Materials, Vol. 23, (2014), pp. 535-550.

Watanabe, J., Tanaka, F., Higuchi, R., Matsutani, H., Okuda, H., and Okabe, T., A study of stress concentrations around fiber breaks in unidirectional CF/epoxy composites using double-fiber fragmentation tests, Advanced Composite Materials, Vol. 27, (2018).

Watanabe, J., Tanaka, F., Okabe, T., Accurate determination of tensile strength distribution for PAN-based carbon fibers, Journal of the Japan Society for Composite Materials, under review. 